\title{
Flexible smart textile coated by PVDF/Graphene oxide with excellent energy harvesting toward a novel class of self-powered sensors: Fabrication, Characterization and Measurements
}

\author{
Nabil Chakhchaoui 1,2,*, Rida Farhan 2, Yu-Ming Chu 3, 4,*, Umair Khan 5, Adil Eddiai 6, Lhaj El \\ Hachemi Omari ${ }^{7}$, Mounir Meddad ${ }^{8}$, Abdelowahed Hajjaji ${ }^{9}$, Omar Cherkaoui ${ }^{1}$, Yahia Boughaleb ${ }^{11}$, \\ Lieva Van Langenhove ${ }^{11}$
}

${ }^{1}$ REMTEX, Higher School of Textile and Clothing Industries (ESITH), Casablanca Morocco; Cherkaoui@esith.ac.ma

2 Laboratoire Nanotechnologies et Nanosystèmes, LN2, CNRS, Université de Sherbrooke,3IT (Institut Interdisciplinaire d'Innovation Technologique), Sherbrooke, QC, Canada; rida.farhan@usherbrooke.ca, nabil.chakhchaoui@usherbrooke.ca

${ }^{3}$ Department of Mathematics, Huzhou University, Huzhou 313000, P. R. China; chuyuming@zjhu.edu.cn

${ }^{4}$ Hunan Provincial Key Laboratory of Mathematical Modeling and Analysis in Engineering, Changsha University of Science \& Technology, Changsha 410114, P. R. China;

${ }^{5}$ Department of Mathematics and Social Sciences, Sukkur IBA University, Sukkur 65200, Sindh Pakistan; umairkhan@iba suk.edu.pk

${ }^{5}$ LPMC, Faculty of Sciences Ben M’Sik Hassan II University, Casablanca, Morocco; adil.eddia@uh2c.ma

${ }^{6}$ Hassan II University, Faculty of Sciences Aïn Chock, LRDYS and LPMMAT, Casablanca, Morocco; bophysiq@gmail.com

${ }^{7}$ LAS Laboratory of Setif, Mohamed el Bachir el Ibrahimi BBA University, Algeria; meddadmounir@gmail.com

${ }^{8}$ Laboratory of Engineer Science for Energy, ENSA, Chouaïb Doukkali University, El Jadida, Morocco; hajjaji.a@ucd.ac.ma

${ }_{9}$ Chouaib Doukkali University, El Jadida, Morocco; yboughaleb@yahoo.fr

${ }^{10}$ Centre for Textile Science and Engineering, Department of Materials, Textiles and Chemical Engineering, Ghent University, Belgium; lieva.vanlangenhove@ugent.be

* Correspondence: chuyuming@zihu.edu.cn; nabil.chakhchaoui@usherbrooke.ca

\begin{abstract}
Because of some of their diverse benefits, intelligent textiles have attracted a great deal of interest among specialists over the past decade. This paper describes a novel approach to the manufacture of intelligent piezoelectric polymer-based textiles with enhanced piezoelectric responses for applications that extract biomechanical energy. Here we report a highly scalable and ultrafast production of smart textile piezoelectric containing graphene oxide nanosheets (GONS) dispersed in polyvinylidene fluoride (PVDF). In this work, Cotton textiles (CT) were functionalized and by graphene oxide (GO), using PVDF as a binder to obtain a CT-PVDF-GO material. Tetraethyl orthosilicate (TEOS) was further grafted as a coating layer to improve the surface compatibility, resulting in the CT-PVDF-GO-TEOS composite. The research results show that the addition of GONS significantly improves PVDF's overall crystallization rate on CT. More specifically, the piezoelectric $\beta$-phase content $(100 \%$ higher $F[\beta]$ ) and crystallinity degree on the piezoelectric properties of composite cotton fiber has been improved effectively. Consequently, this fabricated piezo-smart textile has a glorious piezoelectricity even with comparatively low coating content of PVDF-GONS-TEOS. Based on it, the as-fabricated piezoelectric textile device has resulted in the output voltage of up to $13 \mathrm{mV}$ for a given frequency ( $\mathrm{fm}=8 \mathrm{~Hz}$ ) at fixed strain amplitude value $(0.5 \%)$. It is believed that this research may further reveal the field of energy harvesting for possible applications in the future.. In addition, the set of experimental results that illustrate the smart textile was carried out and discussed, and how it can be used as a wearable device source for this smart textile. Finally, the approach described in this study can also be used to construct other desirable designs, for a wearable low-consumption sensor, etc.
\end{abstract}

Keywords: Smart textile piezoelectric, Graphene oxide, Polyvinylidene fluoride, energy harvesting, self-powered sensors.

\section{Introduction}

Textiles are ubiquitous for us and cover all areas of human activity. They have not only a protective shield or a comforting cocoon, but also an esthetic appeal and a cultural meaning and values. The conventional versatility of textiles has been expanded by recent technologies. Piezo-smart texties for energy recovery are currently attracting great interest. Several conversion modes and energy sources are investigated (electro-mechanical conversion (due to movements and / or vibrations, photoelectric effect or thermoelectric effect, etc.). Different technologies integrate these smart materials into the textile 
structure [1,2]. Non-woven textiles, knitting, embroidery [3,4], braiding, weaving [5,6], coating / laminating [7], printing [8], spinning [9] and chemical treatments [10] are among those that provide unique characteristics, such as regulated hydrophobic actions. PVDF and polymers from the same family have received significant attention in recent decades in terms of intelligent uses, mainly because of their excellent ferroelectric and piezoelectric properties as well as excellent chemical resistance and favorable formability. PVDF has the most common polymorphs of four crystalline phases: $\alpha, \beta, \lambda$, and $\pi$ [11] that make it a semi-crystalline material. The $\alpha$ phase with TGTG0 (trans-gauche) conformation is the most dominant form due to its highly crystalline nature, while the $\beta$ phase with TTTT (all trans) conformation is the most technically appropriate polymorph due to its electroactive features. In addition, the integration into the PVDF matrix of functional nanofillers such as barium titanate, zirconium titanate (PZT), carbon nano fillers, graphene oxide (GO), titanium (TiO2) is an effective and cost-effective method for the induction of $\beta$-polymorph. [12-14]

The integration of intelligent materials in textile systems provides the opportunity to create textiles with a new type of behaviour and function; Power generation or storage [15], human interface elements [16], radio frequency (RF) functionality, or assistive technology [17] could be the active functionality. Wearable sensors provide a sustainable solution through leg movements for energy harvesting technologies, and other research teams are exploiting body heat.

Tide has been created by a structure-based piezoelectric polymer fiber capable of harvesting natural energy, including sun, rain, wind, wave, and research teams from the University of Bolton [18]. The textile will also be ready to execute energy generation operations in addition to behaviors such as sensing [19-21], actuating and conducting electricity [22-24]. Energy harvesting devices using bio materials and ceramic materials were also reported recently [25-29].

Particular attention is paid to the definition of materials and the methodology for the creation of smart electroactive textiles in this work. Intelligent textiles can be divided into two classes: active smart textiles and passive smart textiles. The laters could be employed in a textile structure hence providing an additional function in the passive type, that is, regardless of the changing environment. For instance, they can detect and turn signals into electric ones and they can be used as sensors. However, Active smart textiles are materials capable of adapting their functionality to changes in the environment; in other words, they can automatically sense and respond to environmental conditions or such stimulation. This makes them promising candidates for actuator and sensor devices. In addition, these materials are classified into two categories: (I) the first includes those which modify one or more of their properties in direct reaction to a stimulus and (ii) the second class includes those which reversibly convert the energy of 'a source of energy in other forms of output energy. For example, active smart textiles with shape memory, chameleonic ..., etc [30-32].

In the REMTEX laboratory at ESITH, a pretreatment of cotton-based textiles (CT) was carried out after having treated these textiles in several stages. The choice of cotton fiber was in accordance with the objective of the research. This article proposes a methodology aiming to transform textiles from passive gender to dynamic textiles, ie static functional textiles with dynamic characteristic elements. The example is the $\mathrm{CT}$ will be transformed from a passive textile (without function) into a piezoelectric (dynamic) textile. on the basis of Polyvinylidene fluoride (PVDF) - Graphene Oxide Nanosheets (GONS) - Tetraethyl Orthosilicate (TEOS), $\mathrm{Si}(\mathrm{OCH} 2 \mathrm{CH} 3) 4$. An association between a solvent casting process and the solvent $\mathrm{N}, \mathrm{N}$-dimethylformamide (DMF) was performed, and a coating system followed by evaporation was involved in this novel economic and ecologic process.

CT coated with PVDF-GONS-TEOS nanocomposites is prepared in this article, where approximately $100 \%$ of the electroactive phase is achieved, which is useful for efficient mechanical energy harvesting and performance-enhanced sensing. A piezoelectric nanogenerator (PNG) was being tested to have an output voltage of $13 \mathrm{mV}$ at a fixed strain amplitude value $(1.5 \%)$ on account of the superior electroactive properties of the smart textile. These findings have opened up exciting opportunities for the use of textile fiber-based nanocomposites in different technological applications. It is hypothesized based on a literature survey, that this novel piezoelectric smart textile would help build a flexible, self-powered, multifunctional e-skin for the detection of human motion and a wearable low-consumption sensor source. 
It also addresses various implantable devices that do not need external energy input for applications for in vivo biomechanical energy harvesting or bone defect repair, as well as human activity sensors or detectors, monitoring devices, independent temperature surveillance and pressure management that can be prepared using piezoelectric intelligent textiles.

\section{Materials and Methods}

\subsection{Materials}

Graphite used in this paper was purchased from (LOBA Chemie, ref. "7782-42-5") with a purity of $99.99 \%$, particles size $\leq 20 \mu \mathrm{m}$. Poly (vinylidene fluoride) or PVDF, in powder form, (Alfa Aesar, ref. "24937-79-9"), N, N-dimethylformamide (DMF) (Carlo Erba).. The precursor usually used in the production of composite polymers is tetraethyl orthosilicate (TEOS), [ $\mathrm{Si}(\mathrm{OCH} 2 \mathrm{CH} 3) 4]$ (LOBA Chemie).

\subsection{Characterizations}

Using Fourier transform Infra-Red (FTIR) spectroscopy (Nicolet iS10 FTIR-ATR spectrophotometer), the neat PVDF and nanocomposite films were first characterized by recording transmittance (\%) scans in the range of 4000 to $800 \mathrm{~cm}-1$. Scanning Electron Microscopy (SEM SH-5000PEDS) leads to examine the nanocomposite morphology and chemical composition. Polarized optical microscopy (POM) images were acquired by 3012 SERIES ACCU-SCOPE microscope to visualize the distribution and dispersion of nanofillers inside the polymer matrix.

\subsection{Synthesis of Graphene Oxide Nanosheets (GONS)}

Briefly, Graphite oxide is fabricated by a modified Hummer process from graphite powder [33,34] (Fig.1). Three key steps have been included in a standard protocol for the preparation of GO.

A quantity of $2 \mathrm{~g}$ of graphite powder with $2 \mathrm{~g}$ of sodium nitrate (NaNO3) dissolved in $90 \mathrm{ml}$ of $\mathrm{H} 2 \mathrm{SO} 4(98 \%)$ in a $1000 \mathrm{ml}$ volumetric flask kept under an ice bath $\left(0-5{ }^{\circ} \mathrm{C}\right)$ with continuous stirring. After stirring for 4 hours, $12 \mathrm{~g}$ of potassium permanganate $(\mathrm{KMnO} 4)$ was added to the suspension very slowly. To maintain the reaction temperature below $15^{\circ} \mathrm{C}$, the addition rate is precisely regulated.

The solution was diluted with a large amount of double-distilled water and stirred for 2 hours. Then, the solution was poured into an ice bath kept for 2 hours at $35^{\circ} \mathrm{C}$. The resulting mixture is maintained at $98^{\circ} \mathrm{C}$ for 15 minutes in a reflux process. After 10 minutes, the temperature was reduced to around $30^{\circ} \mathrm{C}$, which made the solution a brown color. After $10 \mathrm{~min}$ the temperature was lowered to $25^{\circ} \mathrm{C}$ and kept constant for 2 hours. The solution is finally treated with $40 \mathrm{ml}$ of $\mathrm{H} 2 \mathrm{O} 2$ to reduce the residual permanganate ions to soluble manganese ions until the evolution of gas ceases, giving a yellow color. In the prepared solution, $200 \mathrm{ml}$ of water was added and stirred for 1 hour. It then settles for 3-4 hours without stirring. The latter was washed several times with $\mathrm{HCl}(10 \%)$ then with deionized water (DI) several times until neutralization $(\mathrm{pH}=7)$.

The gel-like material is vacuum-dried at $60^{\circ} \mathrm{C}$ overnight after centrifugation to obtain GONS (See Fig.1). 
Step 1: Graphite oxide preparation process
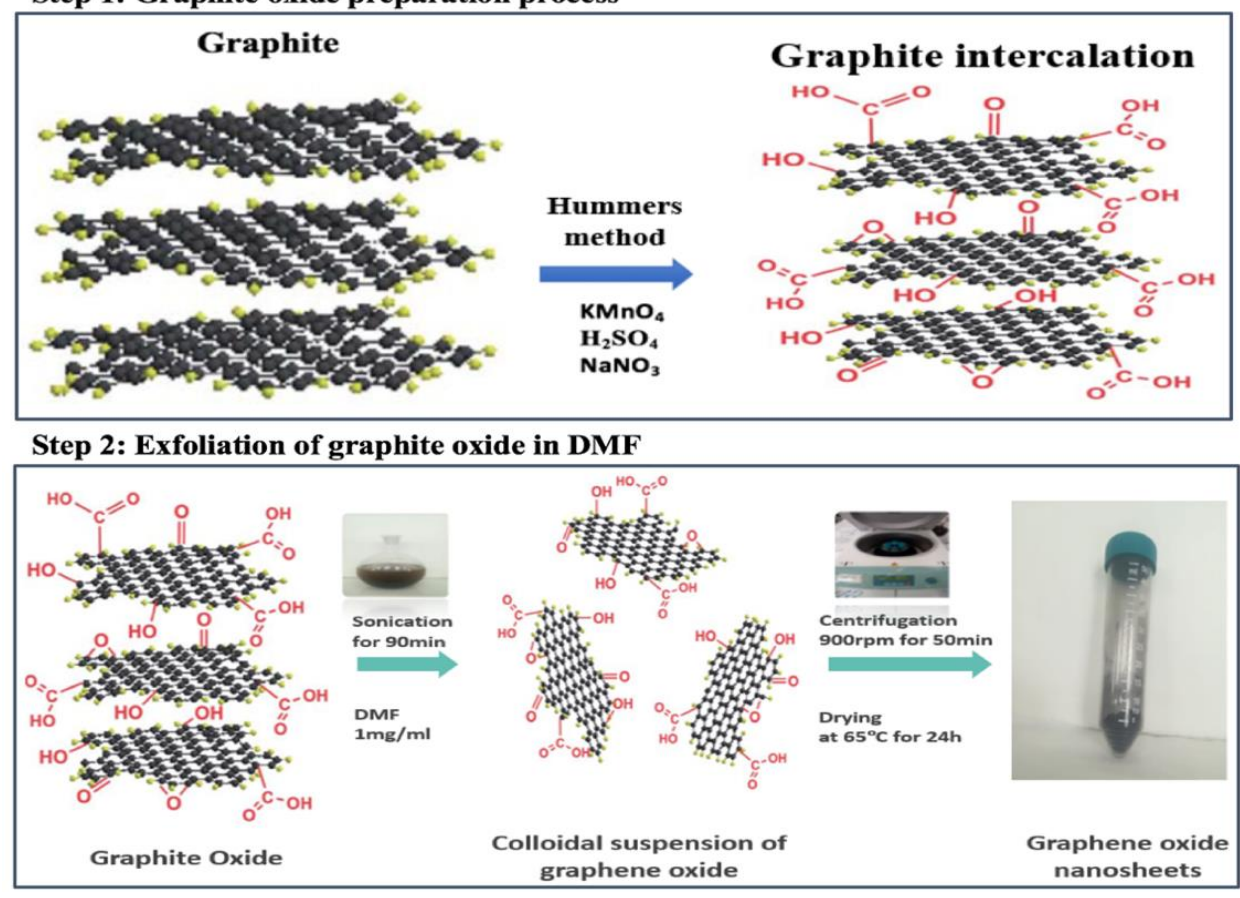

Figure 1. Diagram of the preparation of graphene oxide.

\subsection{Characterization of Graphene Oxide}

The graphene oxide synthesis was carried out by introducing the natural graphite in the presence of an oxidizing agent in a concentrated acid.

The approach of The Hummer has proven that it is a less risky and more reliable technique for graphite oxidation. The most widely used methods for the oxidation of graphite are actually this version and its updated variants [35-37]. Indeed, the preparation of graphene oxide nanosheets was performed through the updated hummer method in this present work. During this process, the surfaces and edges of the graphite structure have been decorated with hydroxyl, carbonyl, epoxide and peroxide groups [38,39]. GO can be stabilized electrostatically to produce a colloidal suspension in water [40], and some organic solvents [41,42] without surfactants, namely DMF, due to the ionization of the carboxyl groups which are mainly present on the edges of the sheet. Ultrasonic agitation and rapid heating facilitated the exfoliation of graphite oxide into the individual sheets $[43,44]$.

Graphite oxidation creates viscous brown slurry, which contains exfoliated sheets and graphite oxide, as well as traces of non-oxidized graphite particles and oxidizing agents in the reaction mixture. Salts and ions from the oxidation phase can be separated from GO suspensions through continuous centrifugation, sedimentation or dialysis [45]. Unoxidized graphitic particles and dense platelets of graphite oxide are precipitated by more centrifugation to achieve a monolayer GO suspension. By density gradient centrifugation, GO flake suspensions that are mono-dispersed depending on their lateral size can also be obtained [46].

In the following section, exfoliated graphene oxide in DMF was used as a nucleating charge for the PVDF semi-crystalline thermoplastic polymer.

As previously indicated, by the complete exfoliation of graphite oxide, nanosheets of graphene oxide were collected through a strong sonification in DMF. The suspension acquired was removed by high-speed centrifugation to isolate a solid phase of graphene oxide, followed by air-drying. The X-ray diffraction pattern (XRD) of graphite $(G)$, graphite oxide (GO and exfoliated graphene oxide (GONS) crystalline structures is shown in Fig.2 (a). To confirm the complete synthesis of GONSs through the modified Hummer process, the X-ray technique was used. The X-ray spectrum of exfoliated GONS shows the absence of a diffraction peak in comparison with graphite $\left(2 \theta=26.23^{\circ}\right)$ and graphite oxide $\left(2 \theta=10^{\circ}\right)$. This indicates that the periodicity of the exfoliated graphene oxide structure has been removed; the findings reported are in good accordance with the literature [47-50]. 
Fig.2(b) shows the infrared spectra of graphite and GONS. On the GONS spectrum, new characteristic bands such as $\mathrm{C}-\mathrm{O}, \mathrm{C}=\mathrm{C}, \mathrm{C}-\mathrm{H}$ and $\mathrm{OH}$ groups have appeared. Using this technique, we have been able to determine the chemical nature of the oxygen-containing functional groups in the graphene oxide structure that are formed between the layers and at the edges of each layer of graphite during oxidation. Chemical, as well as, the effect of ultrasonic waves generated during exfoliation on oxygen functional groups was well examined.

(a)

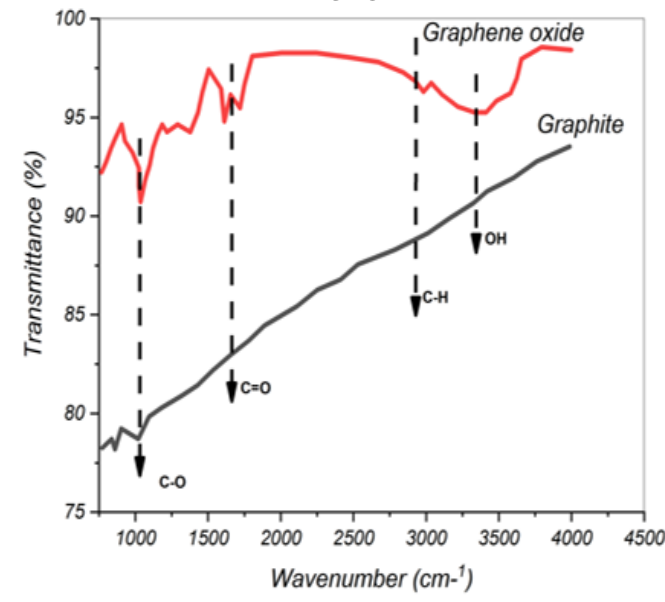

(b)

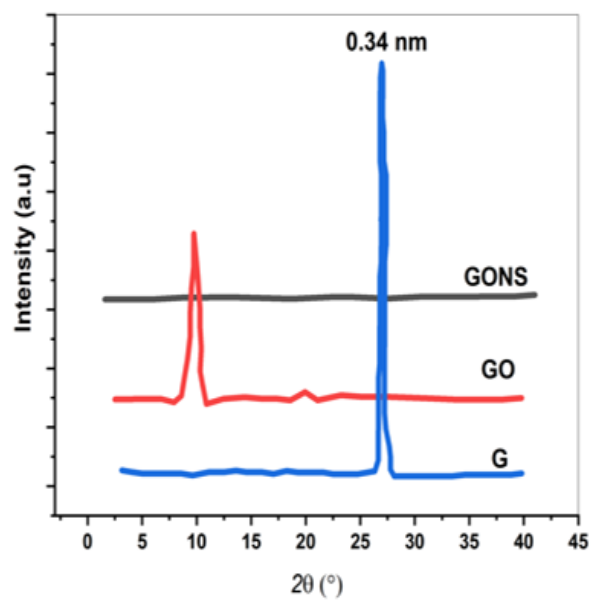

Figure 2. (a) FTIR spectra (b) and XRD patterns of graphene oxide.

The vibration bands at 981 and $1036 \mathrm{~cm}^{-1}$ are assigned to the epoxide group ( $\left.>\mathrm{O}\right)$, the band at 1612 $\mathrm{cm}^{-1}$ is assigned to the $\mathrm{C}=\mathrm{C}$ bond. The intense band at $1713 \mathrm{~cm}^{-1}$ is attributed to the carbonyl elongation vibration (=O). Vibration bands around 2000-3340 $\mathrm{cm}^{-1}$ are attributed to the $\mathrm{C}-\mathrm{H}$ bond. In addition, the bands are disbursed to the hydroxyl groups $\mathrm{O}-\mathrm{H}$ at $3680 \mathrm{~cm}^{-1}$ and $1410-1280 \mathrm{~cm}^{-1}$. Water molecules are attributed to the band at $3650 \mathrm{~cm}^{-1}$. The existence of various transmission bands demonstrates the appearance on the edges and surfaces of each graphene oxide plane of a number of oxygen groups [4750].

The synthesis of exfoliated graphene oxide nanosheets was successfully performed via oxidizing powdered natural graphite by a modified Hummer process. Indeed, the most significant structural changes that occurred during these chemical processes were confirmed by the FTIR technique and reflected in their spectra (Fig.2(b)). The research findings are important and in satisfactory correlation with those mentioned in the literature [51,52].

\section{Processes of Manufacture of Polymer Composite \& Textile Coating}

In this study, Cotton-based knitted configurations have been perfected using a mixing task performed via a solvent coating-evaporation step. This modern approach (economic and environmental) requires the integration of a specific technique for a good mixing of the solutions and a coating approach to manufacture smart textiles from a PVDF matrix and a well-chosen type of nanoparticles., such as graphene oxide nanosheets (GONS) with a TEOS solution.

The deposit formation from a solution containing TEOS on textile surfaces has been extensivly reported in literature [53-56]. However, the presence of silica can aid in coatings to improve the final appearance of the textile, as well as its operating efficiency. Indeed, the addition of TEOS enhanced the solution's grafting onto the cotton textile (CT). As described earlier, using various mass fractions of GONS nanosheets $(0.1,0.5,0.8$ and $1.0 \mathrm{wt} . \%)$, the nanocomposite solution was made. For better solubility of PVDF in the mixture and extreme dispersion of nanofillers during the preparation of PVDFGONS, DMF was used as standard solvent (see Table 1). 
Table 1. Nomenclature of nanocomposite PVDF-GONS-TEOS, compositions and mass proportions.

\begin{tabular}{llccc}
\hline $\begin{array}{l}\text { The Series } \\
\text { Nomenclature }\end{array}$ & $\begin{array}{l}\text { Nomenclature of } \\
\text { Nanocomposites }\end{array}$ & $\begin{array}{c}\text { GONS } \\
\text { Fraction }\end{array}$ & $\begin{array}{c}\text { PVDF } \\
\text { Fraction }\end{array}$ & $\begin{array}{c}\text { TEOS } \\
\text { Fraction }\end{array}$ \\
\hline PVDF-CNC-TEOS & PVDF & $0 \mathrm{wt} . \%$ & $90 \mathrm{wt} \%$ & $10 \mathrm{wt} . \%$ \\
& PVDF- GONS 0,1 wt.\% -TEOS & $0.1 \mathrm{wt} \%$ & $89.9 \mathrm{wt} . \%$ & $10 \mathrm{wt} . \%$ \\
& PVDF- GONS 0,5 wt.\% -TEOS & $0.5 \mathrm{wt} . \%$ & $89.5 \mathrm{wt} \%$ & $10 \mathrm{wt} \%$ \\
& PVDF- GONS 0,8 wt.\% -TEOS & $0.8 \mathrm{wt} . \%$ & $89.2 \mathrm{wt} . \%$ & $10 \mathrm{wt} . \%$ \\
& PVDF- GONS 1 wt.\% -TEOS & $1 \mathrm{wt} \% \%$ & $89 \mathrm{wt} \%$ & $10 \mathrm{wt} . \%$ \\
\hline
\end{tabular}

\subsection{Technique of Solvent Casting}

The solvent casting process is a method which aims to incorporate the particles or the particle / polymer composite into the organic solvent. [57,58]. Thus, after the recuperation of the solvent via evaporation, the nanocomposite is obtained.

The used techniques of stirring are magnetic and sonication aggitations. However, the sonication manner is the most repeatedly used for nucleating agent-based nanocomposite elaboration; it consists of homogenizing and dispersing the constituents. It is well established that the physical and chemical effects of solution sonication on polymers can be both [57,58]. Indeed, in the existence of nucleating elements, the sonic stage has a critical role in the development of polymer chains.

In order to disperse the particles, the selection process of solvent is mostly dictated via the goodness of the polymer that constitut the final matrix, irrespective of these qualities. In other words, for better polymer solubility, a solvent must be found in which the fillers will disperse and reside stable [59]. Therefore, the employment of an acceptable solvent allows the viscosity of the material to be greatly reduced and high-grade composites to be prepared. Evaporation conditions and solvent selection have been shown to have an effect on the uniformity of the final dispersion of fillers in the matrix. [59]. The slow process evaporation phase will cause the particles to re-agglomerate, which is why other process as coprecipitation, spin-coating, drop-casting or pad-dry have been used. The polymer chains coat the fillers during precipitation and thus evade agglomeration.

The solvent casting technique is widely used for the elaboration of polymeric nanocomposites, namely PVDF and its derivatives. The production protocol presented below is described for the preparation of a nanocomposite with the aid of PVDF via solvent casting technique. But it can be generalized to other types of matrices.

Four key steps will summarize this process:

- Dissolution in the solvent of the PVDF powder.

- GONS dispersion in the solvent.

A blend of the two previous alternatives and the inclusion of TEOS.

- Stirring the mixture until the solution becomes viscous and homogeneous.

A graphene oxide nanosheets (GONS) based nanocomposite were prepared under the same processing conditions as illustrated in figure 3. 


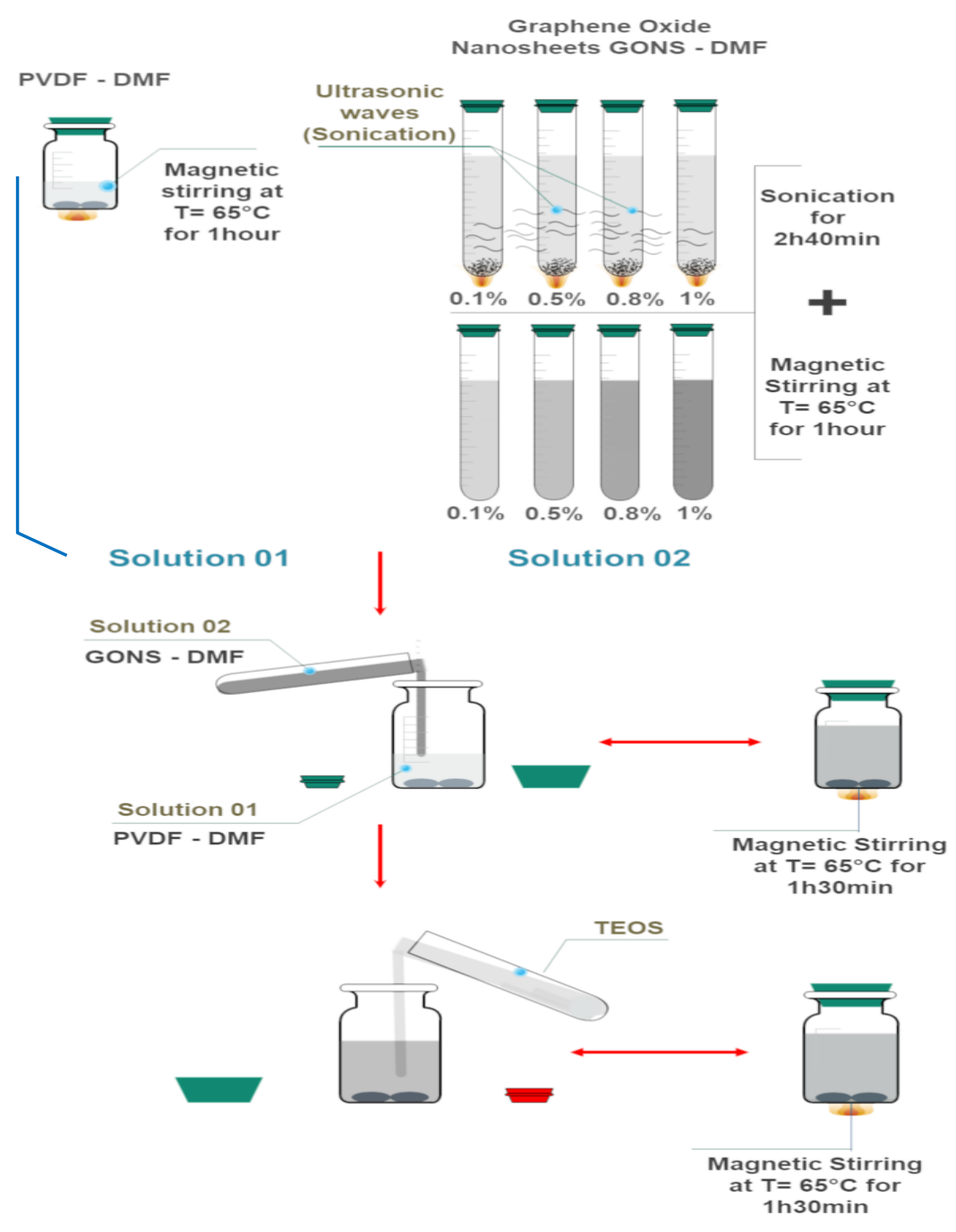

Figure 3. Schematic Representation of PVDF-GONS-TEOS composites synthesis procedure.

\subsection{Cotton Textile Impregnation and Padding through the Nanocomposite Solution}

The "pad-dry-cure" technique outlined in Schema 1 is used to apply solutions to textiles. This impregnation technique makes it possible to deposit silica-based nanocomposite solutions on porous substrates.

The textiles were impregnated in the present work using a bath containing PVDF-GONS-TEOS (nanocomposite solution). The textile is taken out of the bath and the textile is expressed between two compression rollers to ensure the correct dispersion of the solution on the surface until it reaches an $80 \%$ pick-up and then drying for 2 hours at $65^{\circ} \mathrm{C}$, and the pressure applied is 4 bar, the scarf speed is $5 \mathrm{~m} / \mathrm{min}$. 


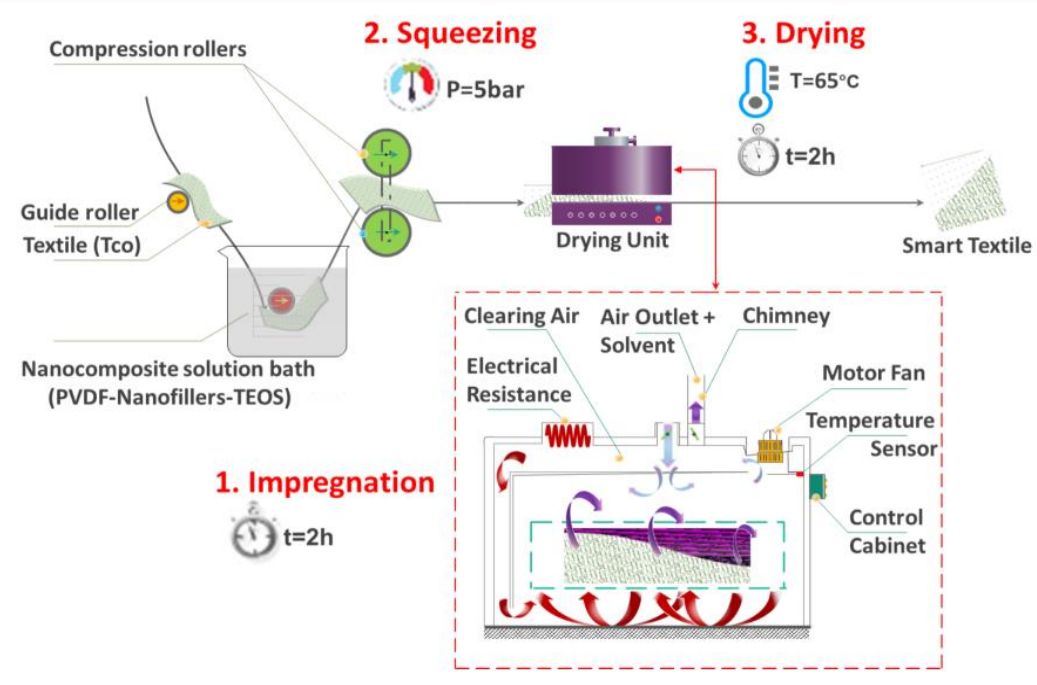

Scheme 1. Schematic illustration of the coating process.

\section{Results and Discussion}

This paper is the subject of the study of the system CT/ PVDF-GONS-TEOS system that is gained by solution casting preceded by a coating process.. Nanocomposite samples are cited in this paper by the symbole "CT / YX-TOES" where CT represent cotton textile, Y represents the PVDF matrix and $X$ has the mass fraction incorporated into the PVDF matrix $(X=0.1 ; 0.5,0.8,1.0 \mathrm{wt} . \%$ graphene oxide nanosheets GONS.

The structure of these textile materials was evaluated by FTIR spectroscopy and EDX analysis coupled with SEM microscopy. The surface morphology of the textile matrices was also studied by optical microscopy (OM) and by SEM.

\subsection{Morphological Properties of Textile Matrices}

Two techniques are widely used to visualize the morphology of textile materials: optical OM and SEM. They include complementary details on the dispersion of nanocomposites within the textile system.

PVDF-GONS-TEOS-based nanocomposites-coated CT was shown in Fig.4 (a-c). Fig. 4 (a-b) shows that the distribution of GONS on the CT is homogeneous.

In this research, optical microscopy was used in this nanocomposite-based textile matrix to illustrate the coating of CT nanocomposites and also the relatively homogeneous dispersion of nanofillers. There is an aggregation of nanofillers at the surface of the textile (non-homogeneous morphological comportment) as is exposed in Fig.4(c) when the nanofillers proportion reaches $1.0 \mathrm{wt} . \%$. The rheological elements of the percolation phenomenon are possibly related to this observation. In the existence of a large number of nanofillers, The nanofillers' aggregation results are expected to be attributed to the limited sonic time.
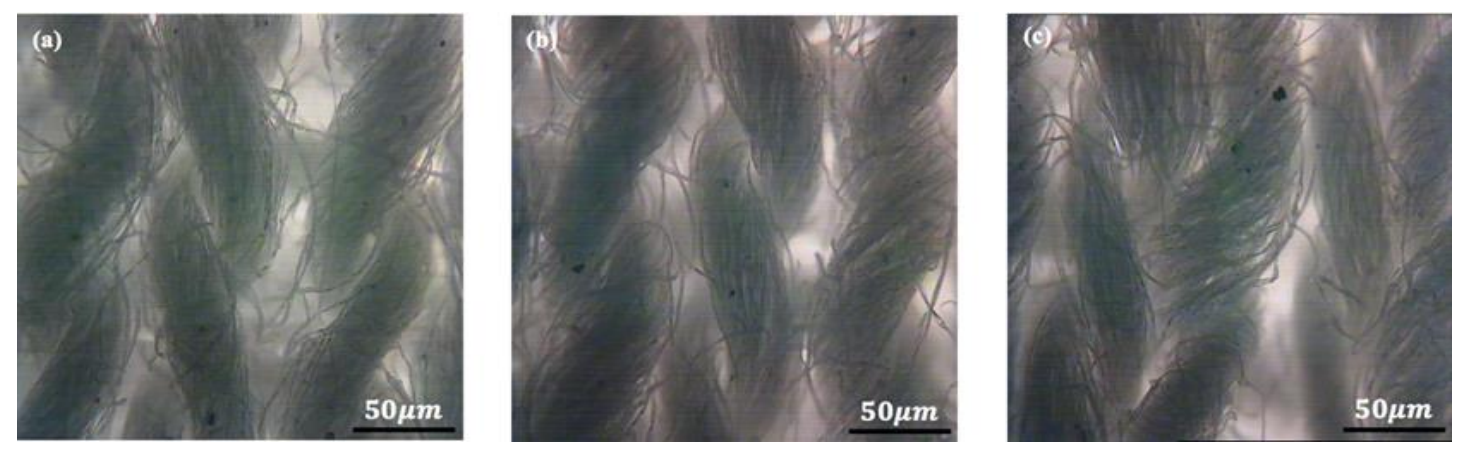

Figure 4. Optical microscope pictures of CT, (a) coated with PVDF-GONS 0.5 wt. \%-TEOS, (b) coated with PVDFGONS 0.8 wt. \%-TEOS, and (c) coated with PVDF- GONS 1.0 wt. \%-TEOS. 
In order to study the morphology of the coatings deposited on the cotton textile indicated previously, SEM observations have been realized. Scanning electron microscopy is based on electron-matter interactions. It allows obtaining high-resolution images of the sample surface with field depths greater than optical microscopy. The typical morphology of cotton textile CT/ PVDF-GONS is described in Fig.5.

Preliminary SEM results demonstrated a random network of overlapping fibers attached to the smooth surface of cotton. There were significant changes between the surfaces of the textile. In fact, the surface of the CT coated with PVDF-GONS $1 \mathrm{wt} \%$-TOES desplays a certain degree of surface irregularity (Figure 5(c)), which disappears in the CT coated with PVDF-GONS 0.1 wt.\% -TOES and 0.05 (Figure 5(a) and 5(b)), due to the compatibility of CT-TEOS and the affinity of the two materials with PVDF. The existence of GONS has significantly influenced such morphology, leading to a high number of distributed particles and bulkier clusters composed by too smaller particles on the surface of fiber (Figures 5(b) and 5(c)), corroborating the results of OM.

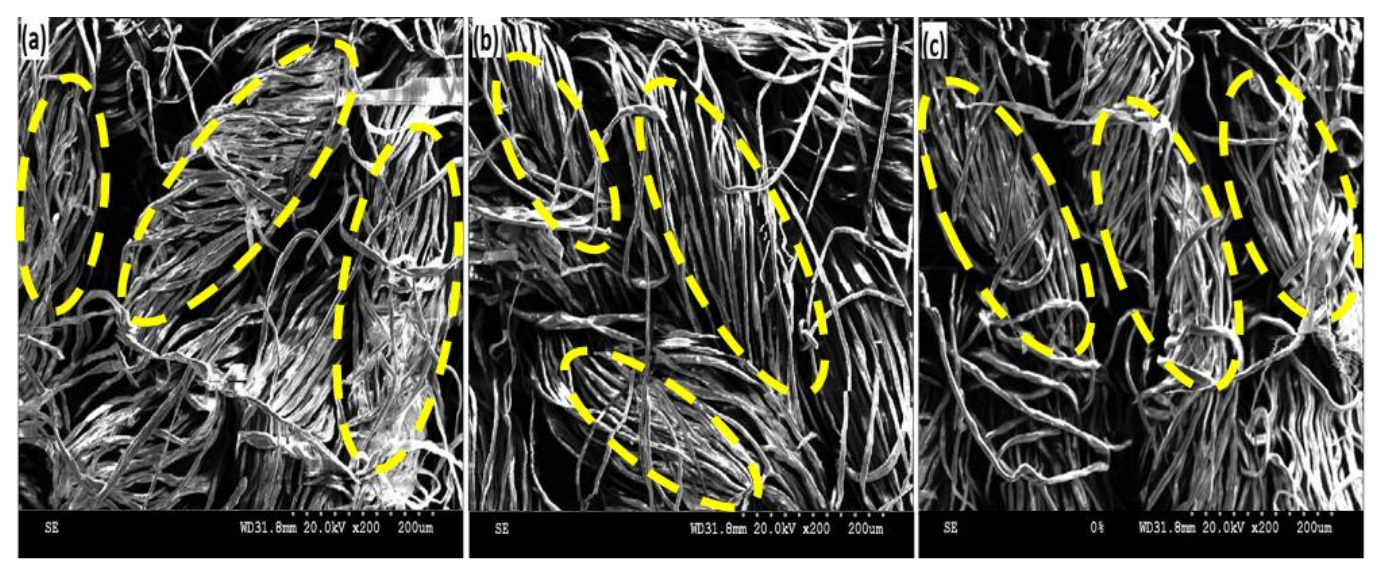

Figure 5. SEM pictures of CT after coating: (a) PVDF-TEOS, (b) PVDF-GONS 0.5 wt.\%-TEOS, and (c) PVDFGONS 1.0 wt.\%-TEOS.

\subsection{Crystalline Phase Characterization}

The crystal structure was evaluated after examination of the morphology and surface structure of fibers. In the Cotton Textiles (CT) analysis, the the phase transformation and the creation of thepurepiezoelectric $\beta$-phase are then confirmed by FTIR measurments based on the transmission bands for both phase $\alpha$ and $\beta$. Results of FTIR spectra for nanocomposite coated smart textiles PVDF-GONS-TEOS are shown in Fig.6.

The structure of the CT was often spécified by IR bands in the region (TCo) 1150-950 $\mathrm{cm}^{-1}$, ascribed to the stretching vibration of $\mathrm{CH}, \mathrm{C}-\mathrm{O}$, according to FTIR results (Fig. 6). In addition, the absorption peaks associated with $\mathrm{OH}$ bands have disappeared at $1204,1236 \mathrm{~cm}^{-1}$, affected to $\mathrm{CH}$ stretching vibration at $1308 \mathrm{~cm}^{-1}$, and the large ones associated with 998-1002 $\mathrm{cm}^{-1}$ and $1456 \mathrm{~cm}^{-1}$ linked to C-O stretching vibrations [60,61]. A new band at $869 \mathrm{~cm}^{-1}$ assigned to $<C F 2$ vibration for amorphous PVDF was created by PVDF incorporation. In addition, the hybrid material is polymerized on the surface of textile during the drying process. This contributes to the formation of covalent bonds of the cotton textile (Si-O-fiber) type, resulting in the emergence of a new characteristic band of Si-O-Si groups at $1063 \mathrm{~cm}^{-1}$ [62]. The fiber profile of the cotton was successfully preserved. It is anticipated that treated CT can interact with PVDF-GONS-TOES functionalized species through sonication via O-bridges. These data findings allow us to expect that the coated PVDF-GONS-TEOS has been successful deposited on the textile surface.

In this figure, the textile shows intense bands at 1210, 975 and $855 \mathrm{~cm}^{-1}$ which are associated to the pure $\alpha$ phase formation [63]. For CT/ PVDF-GONS $0.1 \mathrm{wt}$.\%-TEOS, the infrared spectra show additional peaks to those observed in pure PVDF (Fig.6). These peaks are located at 1279, 1234 and $840 \mathrm{~cm}^{-1}$ is associated with the $\beta$ phase [64-67]. 
The obtained results corroborate the presence of a phases mixture between the $\alpha, \beta$ and $\gamma$ in CT/ PVDF-GONSTEOS cotton textiles containing small mass fractions of GONS (0.1 wt.\%). On the other hand, the textiles containing mass fractions (PVDF-GONS 0.5 wt.\%-TEOS, and PVDF-GONS 0,8 wt.\%-TEOS), only the characteristic peaks of the $\beta$ phase are present $\left(1279,1234\right.$ and $\left.840 \mathrm{~cm}^{-1}\right)$. Also, all specific peaks of the $\alpha$ phase (1210, 975 and $855 \mathrm{~cm}^{-1}$ ) have almost disappeared, which indicates the success of total transformation of the $\alpha$ phase to the $\beta$ one by adding only $0.5 \mathrm{wt} \%$ of GONS.

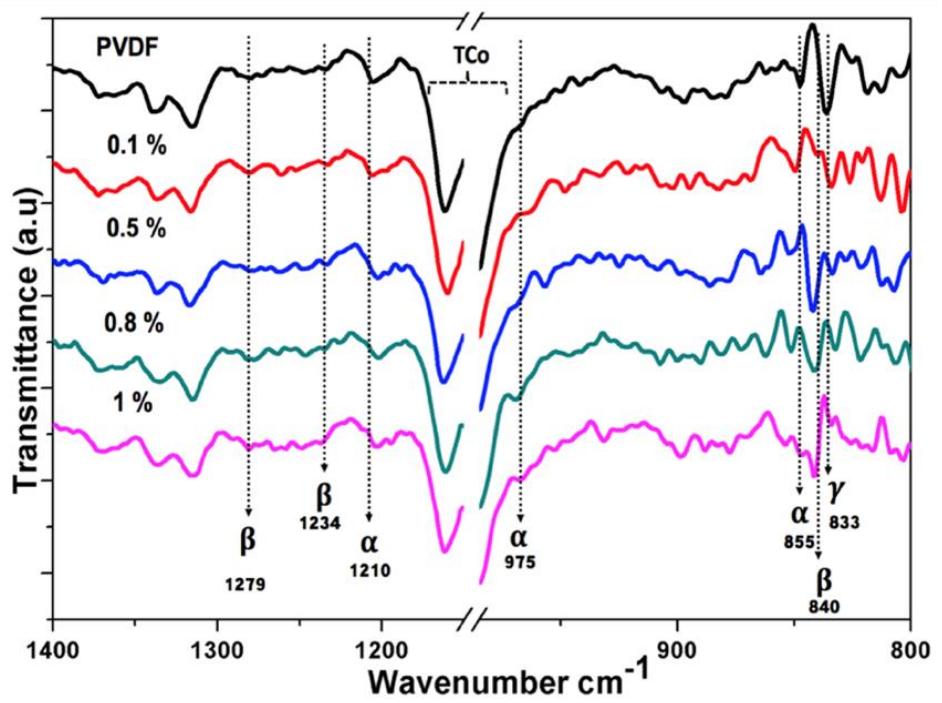

Figure 6. Infrared Spectra (FTIR) of cotton textiles (CT) based on PVDF GONS-TEOS at different mass fractions of GONS.

To confirm the successful preparation of CF/ PVDF-GONS-TEOS, we used EDX-SEM analysis. As shown in Fig.7, spectra of the elemental fluorine (F) and silica (Si) had been observed. The elemental (F) and (Si) observed in all spectra of CF-PVDF-GONS-TEOS provides evidence of the presence of PVDF and TEOS in these materials. Carbon $(\mathrm{C})$ and oxygen $(\mathrm{O})$ atoms were also observed in all EDX-SEM spectra, demonstrating the presence of various functional groups in the nanocomposite structures. This provides strong for the role of TEOS which is beneficial for stabilization and dispersion of PVDFGraphene oxide nanosheets on the surface of CT.
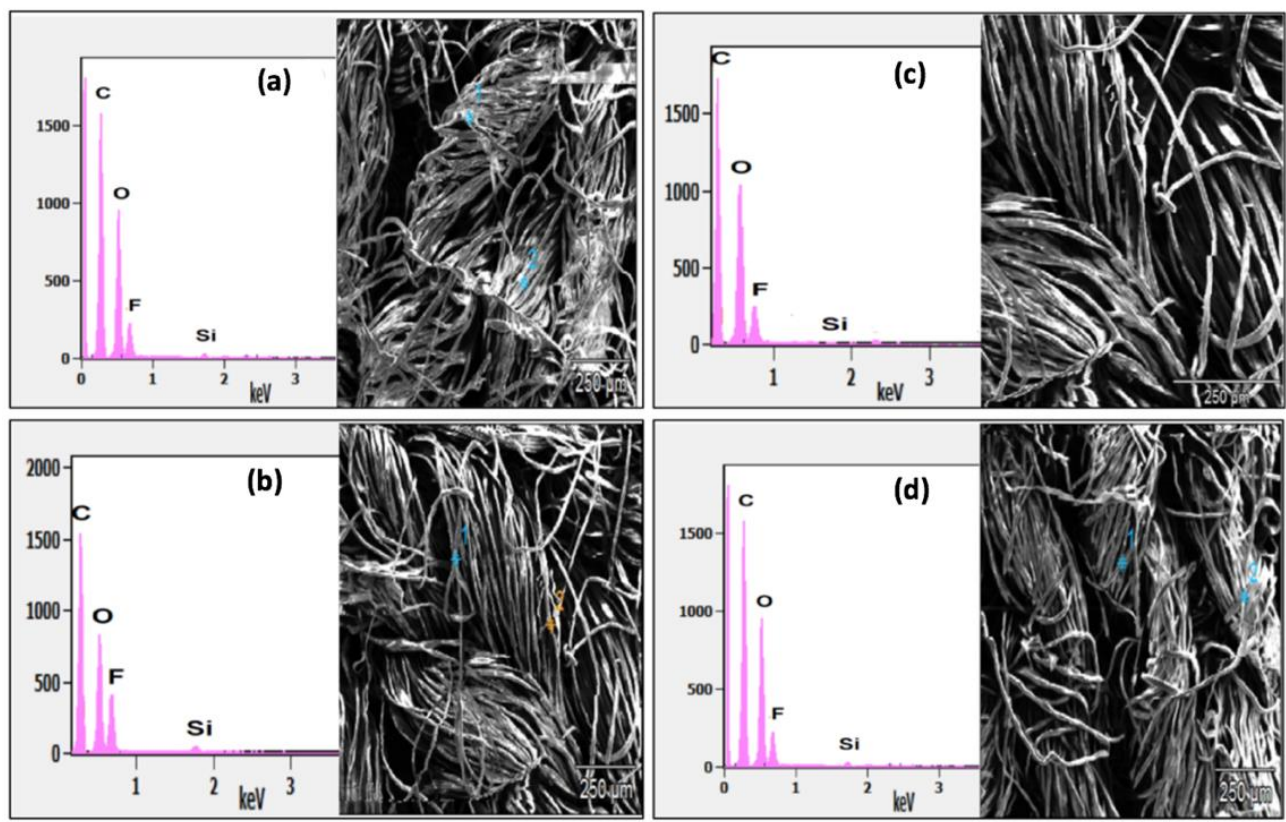

Figure 7. EDX-SEM analysis of cotton textiles (CT): (a) coated with PVDF-TEOS, (b) coated with PVDF-GONS 0.1 wt.\% - TEOS, (c) coated with PVDF- GONS 0.5 wt.\% - TEOS and (d) coated with PVDF- GONS 1.0 wt.\% - TEOS. 


\subsection{CT/ PVDF-GONS Interactions}

Poly (vinylidene fluoride) (PVDF) is one of the most attractive polymers because of its remarkable pyroelectric, piezoelectric and ferro-electro-active properties [68-71]. These characteristics result from its unique polymorphism, which gives rise to exceptional mechanical characteristics, high thermal properties, chemical stability and biocompatibility [72].

Of the four significant crystalline phases $\alpha, \beta, \gamma$ and $\delta$ [69-73] (Fig.8); the electro-active $\beta$ phase is the most used for detection, actuation [74,75] and micro-generation applications [71,76,77]. Various methods of crystallization of the $\beta$ phase have been studied, in particular melt casting [82], solution deposition [78,79], spin coating $[79,80]$ and phase inversion [81,82]. Whereas the PVDF films formed by fusion/crystallization are dominated by the $\alpha$ [83] phase, those obtained by coating by centrifugation and dried at temperatures between 30 and $60{ }^{\circ} \mathrm{C}$ are mainly dominated by the $\beta$ phase [80]. During phase inversion, PVDF films are formed by rapidly quenching the cast films in a solvent-free bath to induce liquid-solid and liquid-liquid phase separation events [72,81,82].

The microstructure and the crystalline phase are controlled by adjusting parameters such as composition [72, 80], type of solvent [84], quenching temperature [85], etc. While the most common method for inducing the $\beta$ phase is the incorporation of nanosheets into the PVDF matrix at an appropriate temperature. Currently, the easy preparation of cotton textiles (CT) based on beta-phase ( $\beta$ ) self-polarized PVDF- GONS -TEOS nanocomposites and with a high coefficient of piezoelectric resistance for electro-active applications has been well reported.

The formation of the $\beta$ polymorph in CT/ PVDF-GONS textiles is attributed to the presence of strong and specific interactions between the carbonyl group $-\mathrm{C}=\mathrm{O}$ found on the surface of the graphene oxide nanosheets (GONS) and the segment $<\mathrm{CF}_{2}$ found in the PVDF. In cotton fabrics coated with PVDFGONS-TEOS, the total formation of the $\beta$ polymorph was observed at the mass fraction of GONS (0.5 wt.\%).

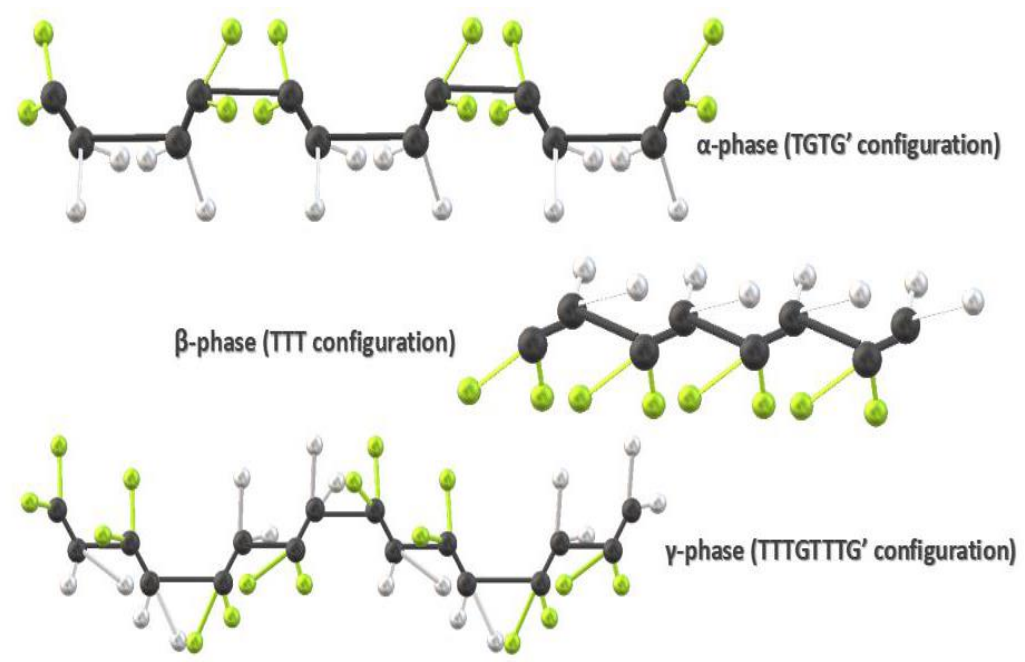

Figure 8. Chain arrangement diagrams for the $\alpha, \beta$ and $\gamma$ crystal phases of PVDF.

\subsection{Mechanism of Formation of the $\beta$-Phase}

The phase composition and morphology of the coated PVDF on CT is strongly affected by the processing conditions. The introduction of nanofillers into the CT-coated PVDF polymer results in the alpha phase $\beta$ transformation. The polymer chains are rearranged in the crystals by this transformation, so that every Trans-plan zigzag conformation (TTT) is caused. This helps the polymer chains' dipoles to match uniformly and thus to form self-polarized crystals. By adding $0.5 \mathrm{wt}$.\% percent GONS to the PVDF polymer via the solution stirring method followed by coating on $\mathrm{CT}$, the $\beta$-phase content maximum value was obtained. The percentage of the $\beta$ phase in textile (CT) coated with nanocomposite (PVDF-GONS-TEOS) can be determined from FTIR analysis using the following equation [86].

$F(\beta)=\frac{A_{\beta}}{1.26 A_{\alpha}+A_{\beta}} \cdot 100$ 
With $\mathrm{A}_{\alpha}$ and $\mathrm{A}_{\beta}$ are the absorbance corresponding to $855 \mathrm{~cm}^{-1}$ and $840 \mathrm{~cm}^{-1}$ bands, respectively, in the FTIR spectra.

On textiles (CT) processed in the $4000-800 \mathrm{~cm}^{-1}$ wavenumber range, FTIR measurements were reported (See Fig.6). The FTIR spectrum of transmission (T) is shown in Figure 6. The percentage of the $\beta$ phase was assessed from this FTIR spectrum using the formula linked transmittance and absorption: $\mathrm{T}+\mathrm{A}=1$.

Consequently, in cotton fabrics coated with PVDF-NFOG 0.5 wt. \% -TEOS the proportion of the $\beta$ process is equal to $100 \%$. This percentage, as shown in Fig.6, amounts to the complete disappearance of the $\alpha$ phase transmission band at $855 \mathrm{~cm}^{-1}$. On other side, for the textile CT/ PVDF-GONS $0.1 \mathrm{wt} . \%$ TEOS, a percentage of $\beta$ phase up to $45 \%$ was determined. Such data analysis reveals the existence of the $\beta$ phase and the $\alpha$ phase mixture in the textile-based nanocomposites PVDF-GONS-TEOS.

Therefore, from the experimental and the calculations data acheived using the above equation (Eq. (1)), it is clear that $100 \% \beta$-phase formation was acquired by adding only $0.5 \mathrm{wt} . \%$ GONS to the above prepared cotton CT. The complete adsorption of the PVDF molecular chains on the GONS surface in the CT/ PVDF-GONS-TEOS is explained by the creation of pure $\beta$ phase in the PVDF-based nanocomposite CT. This is achieved using the shock waves produced during the mixture sonification (see Fig.8) and the use of polar solvents for instance DMF [87].

To maximize the amount of $\beta$ phase produced, the temperature is maintained below $70{ }^{\circ} \mathrm{C}$ during the evaporation, because temperatures above $70^{\circ} \mathrm{C}$ yield to a mixture of $\alpha$ and $\beta$ phases [88-92].

Consequently, adsorption in $\mathrm{CT}$ is exactly related to the sturdy exchange interaction between the $>$ $\mathrm{CF}_{2}$ group of PVDF and the $-\mathrm{C}=\mathrm{O}$ group exists at the surface of GONS (See Figure 9). As well, the formation of the pure $\beta$ phase in the CT-based nanocomposites PVDF was due to the adsorption of the TT conformation on the surface of GONS (see Fig.9 and Fig 10).

The production of a pure $\beta$ phase in CT observed for a mass fraction of GONS (0.5\%) is clarified by the presence of a nanosheet surface appropriate during the crystallization phase for the adsorption of all PVDF chains [93].

Previous studies have shown that increasing the crystallinity of electroactive materials is one of the main elements in increasing the content of the piezoelectric $\beta$-phase. Lund et al. [94] have identified that PVDF fibers of up to $80 \%$ crystallinity, primarily in the $\beta$ phase, under such conditions of melt spinning and cold drawing. El Achaby et al [95] proposed a new approach to composite graphene oxide nanosheets (GO)/PVDF preparation techniques. This technique made it possible to get the purely piezoelectric $\beta$-polymorph formation at just $0.1 \mathrm{wt}$. $\%$ GO material. A mixture of $\beta$ and $\alpha$-polymorph was remarked beneath the material.

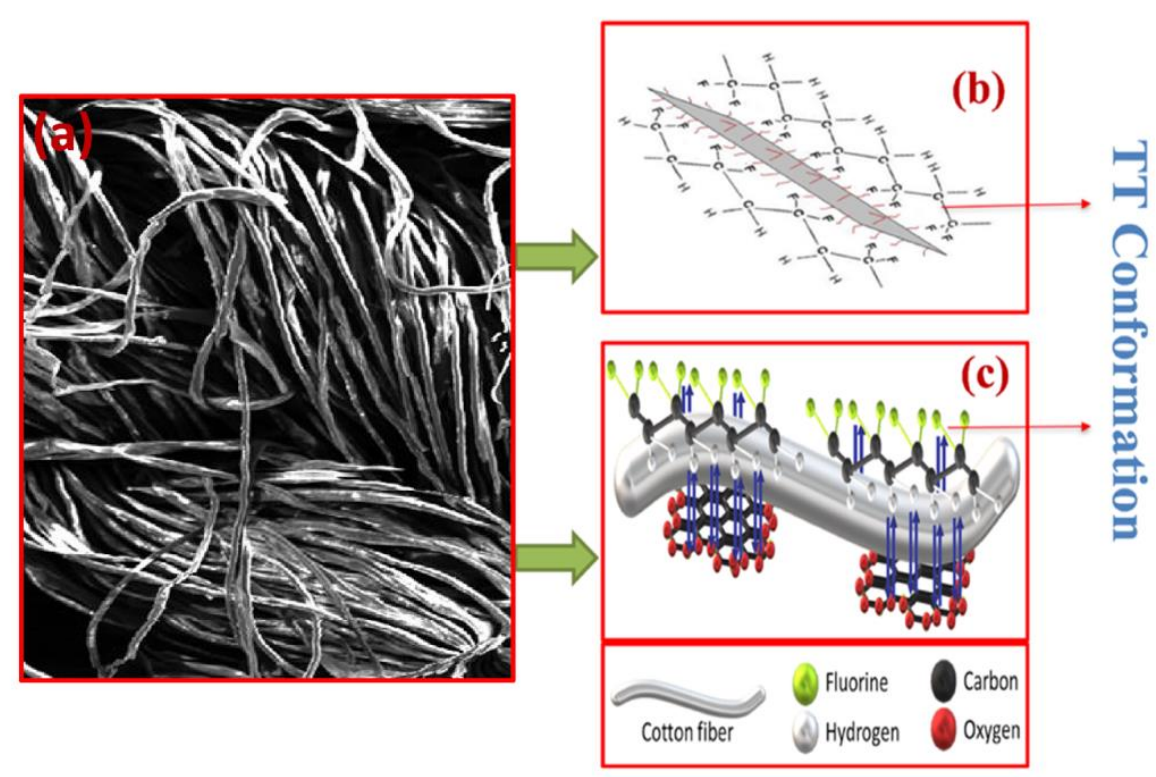

Figure 9. Reaction mechanism described the surface compatibility (a) cotton fibers, (b) PVDF-GONS, and (c) Cotton fiber /PVDF-GONS-TEOS. 


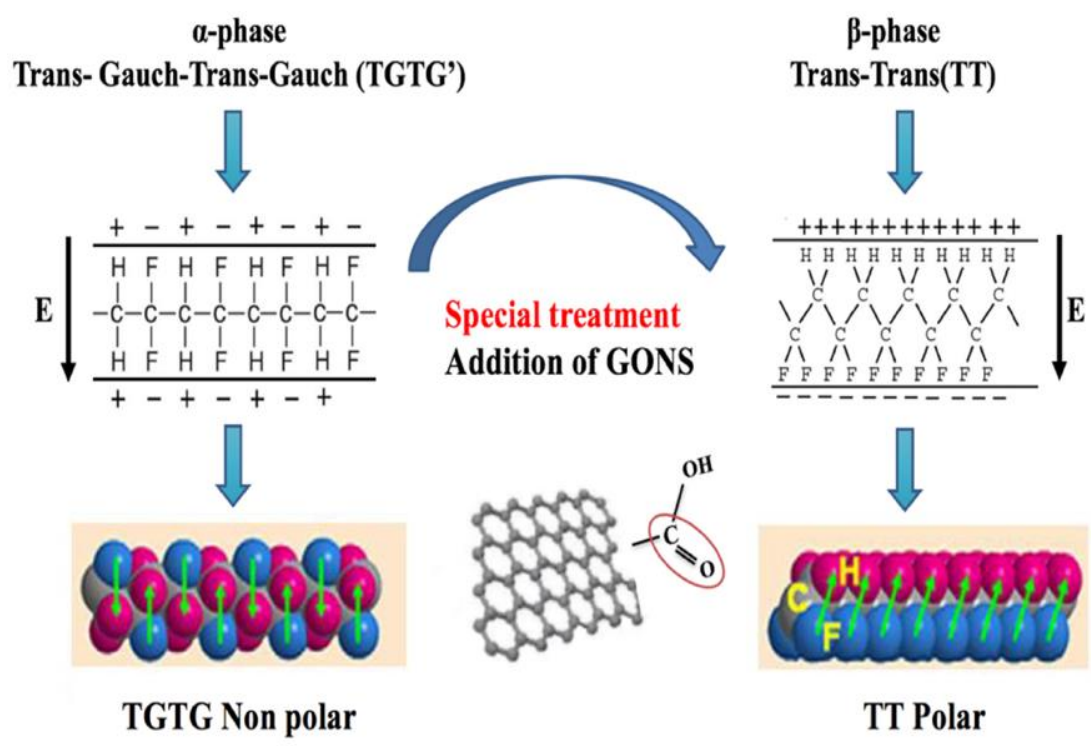

Figure 10. Graphical illustration of the proposed $\beta$ phase transformation mechanism.

\section{Piezoelectric effect for energy harvesting}

\subsection{Working mechanism and device performance}

The smart textile samples (Fig.11) were tested through an electromechanical test rig to prove the structural piezoelectric effects. Fig.12 shows a schematic description of the experimental setup used by the CT/PVDF-GONS-TEOS smart textile to represent the harvested voltage.

This system was established on a test rig with an immobile part and a second part that could be driven in 1 direction with the aid of an ironless linear motor XM550 (Newport Cop., Irvine, CA). Consequently, the textile fiber is stretched at a fixed strain amplitude value $(0.5 \%)$ for a given frequency $(\mathrm{fm}=8 \mathrm{~Hz})$. It was believed to be strained along the 1 direction. The piezoelectric smart textile was connected to an electric charge R to determine the harvested voltage (See Fig.12 (b)).
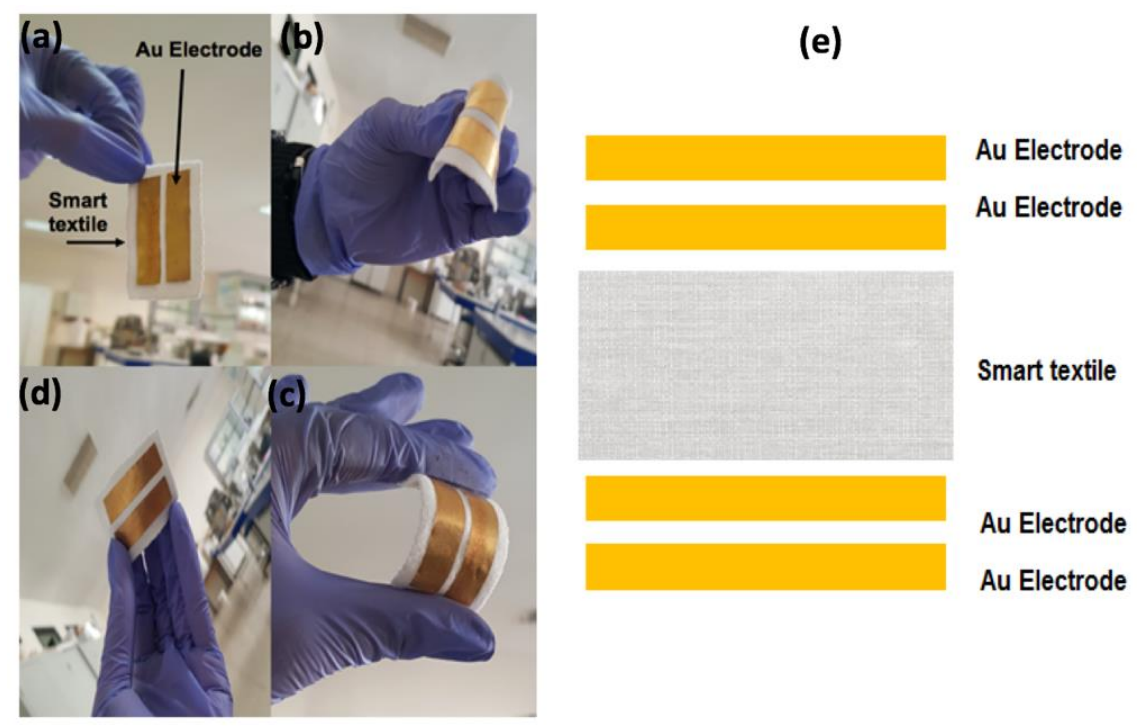

Figure 11. (a, e) Description of the composition of piezoelectric smart textile. (b, c) Bending mode of smart textile. (d) shearing mode of textile. 
(a)

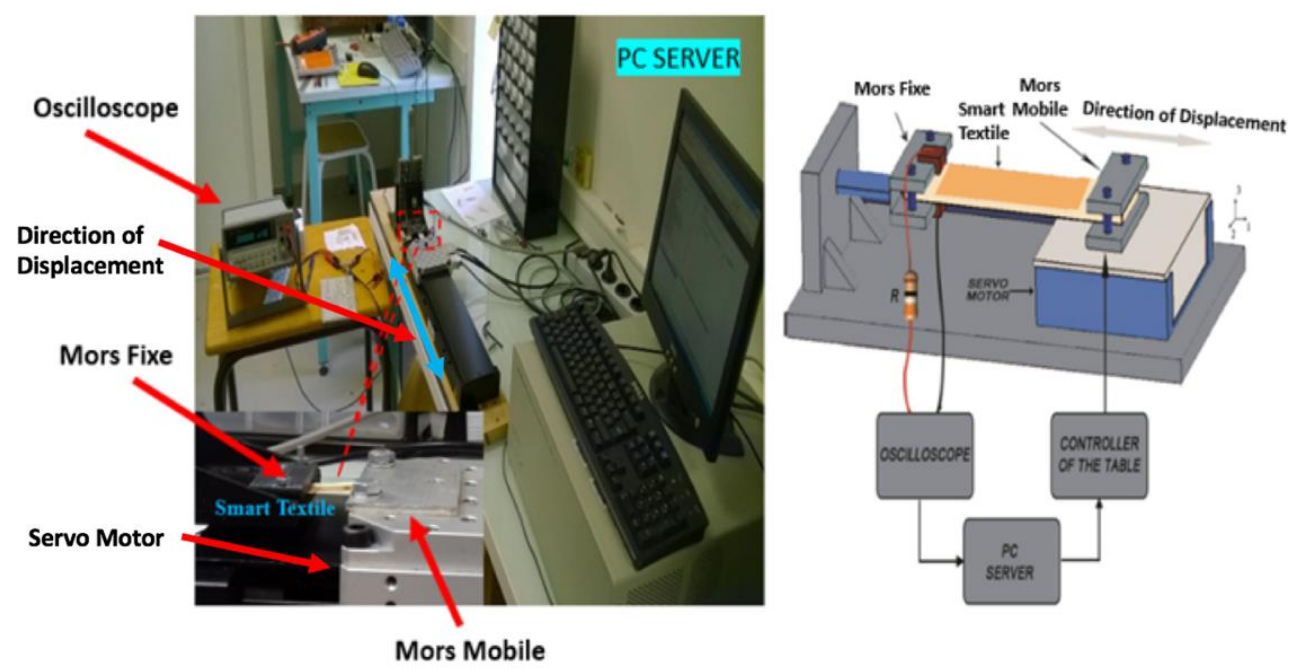

Figure 12. $(a, b)$ Setup and Schematic diagram of the electromechanical setup for the voltage harvesting measurements.

\subsection{Energy Harvesting from Textiles from Stretching Force}

It is well known that at its origin, the piezoelectric effect of materials can be classified to four categories: I the intrinsic piezoelectricity because of the internal strain in the crystal; (ii) the intrinsic piezoelectricity related to the strain-dependence of spontaneous polarization; (iii) the piezoelectricity, on the other hand, resulted from the polarization charge arising from strain-independent persistence; and (iv) Piezoelectricity from the charge embedded in the composite material. However, a number of solutions have been used in the literature to enhance the piezoelectric properties of electroactive materials for energy harvesting, such as the polishing effect at high temperatures [96] or the uniaxial stretching amplitude [97], but also the surface modification of the coating process based on the interfacial contact with the integrated charge. In energy conversion, this last element becomes a critical parameter.

To examine the relationship between volume fraction of charge incorporated and harvested performance of the piezoelectric smart textile, the relative electrical measurements were further performed, and the results of harvested voltage was presented in Fig.11. The output voltage was performed at $8 \mathrm{~Hz}$ for a constant strain of $0.5 \%$ and various external resistive loads (Fig.13 (a)).

From this Figure, the harvested voltage changing with the volume fraction and the variational tendency correctly agree with the above structural characterization of the smart textile. It is clear that the mechanical energy harvesting property depends on the effect of GONS on the sample. Among different volume fractions, the textile gains the best electrical performance when the volume fraction to be 0.5 wt. \%. This phenomenon might be ascribed not only to the abundant GONS particle content but also to the high $\beta$-phase lamellar crystal content. Furthermore, the tendency of voltage evolution with the volume fraction is further analyzed by applying an external strain (1.5\%), as shown in Fig.13. It can be found that, where the volume fraction is $0.5 \mathrm{wt} . \%$, the maximum output could attain up to $13 \mathrm{mV}$. This property exposes the immense measuring potential of mechanical energy harvesting. Furthermore, the detailed results concerning the output voltage varying with the volume fraction are clearly shown in Fig.13 (b). Obviously, when the volume fraction reaches $0.1 \mathrm{wt}$, it is too low to cause the sample's phase transition, the nucleation caused by GONS particles becomes less than the destruction of molecular chains, and the $\beta$-phase content is decreasing than pure PVDF. While, the $\beta$-phase content decreased, the voltage would be composed of the synergistic output produced by the graphene oxide nanosheets. Therefore, there is no distinct decrease in production compared with pure PVDF. Later, the synergistic effect and the amount of $\beta$-phase lamellar crystal are elevated synchronously with the rise in volume fraction, which facilitates a sharp increase in harvested voltage. The best harvested voltage is obtained when the volume fraction exceeds $0.5 \mathrm{wt} . \%$. After that, when the content of GONS nanosheets exceeds the critical point the harvested voltage starts to decline. The probable explanation is that redundant 
GONS particles inhibit the orderly arrangement of the molecular chain, which avoids the formation of the $\beta$-phase lamellar crystal. This result entirely obeys to the original hypothesis of working theory, and the smart textile samples prepared by the method presented possess exceptional piezoelectricity level under the low volume fraction.
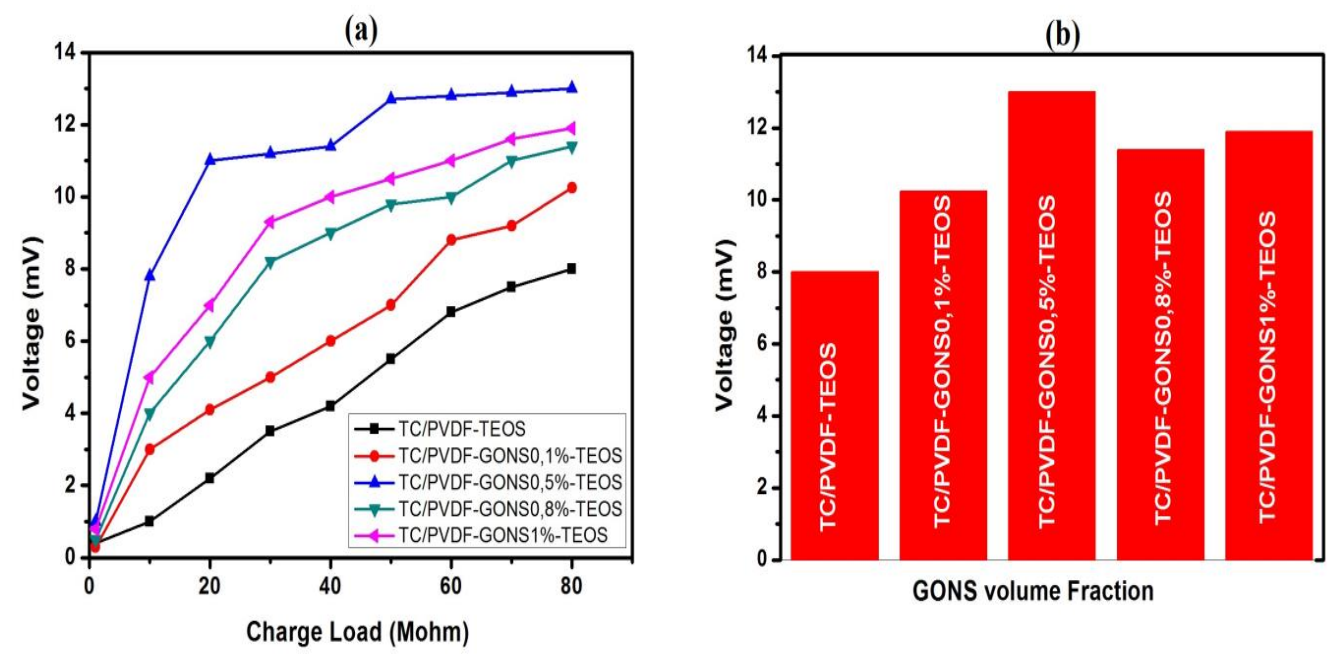

Figure 13. (a) Piezoelectric output voltage values obtained for piezoelectric smart textiles (b) Illustration of the voltage varying with the GONS volume fraction.

During one stretching and releasing action, figure 14 shows the complete features of a piezotextile. The charge centers of the cations and anions correspond with each other at the original and undisturbed state. The positive and negative charges are equally distributed while there is no applied tension on the fabric. Inside the piezoelectric material, no polarization can be observed, so there is no potential difference (Fig. 14(a)). As has been well recognized, piezoelectricity in a non-polar crystal is brought about by the internal strain in the crystal. The internal strain induces the atoms displacement, which is not affine to the deformation of the crystal lattice that is slightly changed. In order to form electrical dipoles, the charge centers are separated and electrical dipole moments change, resulting in the creation of a piezo potential between the electrodes (Fig. 14(b, c)). Electrons flow back to rebalance the charge caused by the pressure released in the short-circuit state when the external force is released (Fig. 14(d)).

Piezoelectricity is also caused by the charge incorporated in the structure of the fabric (the creation of the pure $\beta$-phase in the fabric of the nanocomposites was due to the adsorption of the nucleating agent (GONS) surface TT conformation). The charge imbalance therefore produces a polarization that creates a possible difference.

This result is related to the piezoelectric property of smart textile strongly, therefore, depends on the crystalline structure of the polymer, as well as on the electroactive beta polar phase formation in the textile with the least polar (TC/PVDF-TEOS) demonstrating the lowest harvested voltage and TC/PVDFGONS $0.5 \mathrm{wt} . \%$-TEOS, which is most polar, producing the highest voltage.

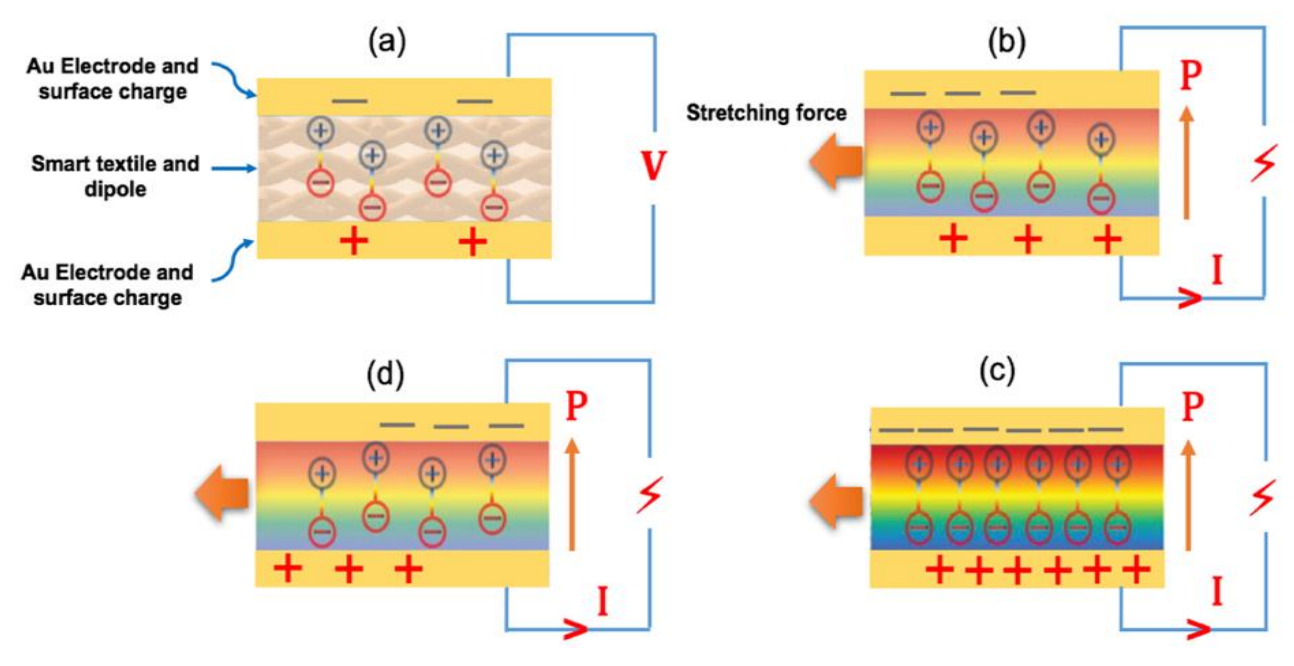


Figure 14. Working mechanisms of piezoelectric textile with schematic illustration of piezoelectric charge generation process.

\section{Conclusions}

In this work, we succeeded in fabricating smart piezoelectric textile through the functionalization of cotton-based textiles (CT). It was carried out using PVDF-GONS-TEOS nanocomposites with filled polar crystal form. By crystallization without applied field or mechanical deformation, the $100 \%$ polar $\beta$-phase was clearly achieved. These textiles have been studied to yield an intrinsic structure, for instance the smart textiles, hence to promote its consideration in the field of textile applications. DMF solvent, as a mixing medium, and TEOS were used to facilitate the grafting of the solution on the cotton textile. These textiles have been functionalized by a novel economic and ecological system, which involves the combination of both process: solvent casting and coating method.

Graphene oxide nanosheets (GONS) were excluded from exfoliation by graphite oxide via sonication treatment in DMF. We find that GONS causes all the trans (TT) conformations that lead to the formation of polar PVDF crystals to be adopted by the PVDF chains. Due to the unique interactions of the carbonyl group $(-\mathrm{C}=\mathrm{O})$ with $>\mathrm{CF}_{2}$ groups in PVDF chains, the advantages of using this method is that these nanofillers (GONS) have great compatibility with the PVDF polymer, ensuing in the standard $>\mathrm{CF}_{2}$ alignment on the GONS surface with all zigzag conformations.

SEM and OM images revealed that nanocomposites of PVDF-GNOS-TEOS were well dispersed at the fiber surface and distributed on the textiles while preserving their appearance and their visual appearance. EDX-SEM analysis, provides evidence of the presence of all chemical elements and demonstrating the presence of various functional groups in the composite structures. FTIR spectra confirmed the phase transformation and the formation of $\beta$-phase in the Cotton textiles.

This research provides new insight into the beneficial role of PVDF as electroactive polymer and GONS as nucleons, i.e. their capability to change CT from a passive textile (without function) to a piezoelectric (dynamic) textile. Yet, in this paper, a mechanism to attain this transformation has been advised. In the textile industry, the proposed treatment process could be used to manufacturing functional smart textiles with high piezoelectric effect. The study provides valuable findings for material production and structure optimization and contributes to the design of harvesting energy equipment and sensor mobiles. The piezoelectric electrical output response of the smart textile was thoroughly studied. It displays an output voltage of $13 \mathrm{mV}$ for a given frequency $(\mathrm{fm}=8 \mathrm{~Hz})$ at a fixed strain amplitude value $(1.5 \%)$.

Unambiguously, these results support the applications of smart textiles in piezoelectric energy conversion as well as in self-powered sensing.

Acknowledgments: The authors would like to express appreciation for the support from the National Natural Science Foundation of China (Grant numbers 11971142, 61673169).

Conflicts of Interest: The authors declare no conflict of interest.

\section{References}

1. Stoppa, M.; Chiolerio, A. Wearable Electronics and Smart Textiles: A Critical Review. Sensors 2014, 14, 1195711992. https://doi.org/10.3390/s140711957.

2. Chakhchaoui, N.; Ennamiri, H.; Hajjaji, A.; Eddiai, A.; Meddad, M.; \& Boughaleb, Y. Theoretical modeling of piezoelectric energy harvesting in the system using technical textile as a support, Poly. for Advan. Techno. 2017, 28, 1170-1178. https://doi.org/10.1002/pat.4010.

3. Moro, R.; Agneessens, S.; Rogier, H.; Bozzi, M. Wearable textile antenna in substrate integrated waveguide technology, Electr. Lett. 2012, 48, 985-987. DOI: 10.1049/el.2012.2349.

4. CMI. Available online. https://www.colonialmills.com/PublicStore/catalog/Braiding-Process, 156. aspx (accessed on 10 October 2020).

5. Textile Innovation $\quad$ Knowledge Available online http://www.tikp.co.uk/knowledge/technology/coating-and-laminating/laminating (accessed on 10 October 2020). 
6. Custom Fabric Printing. Available online http://sophiasdecor.blogspot.it/2012/09/insidespoonflower-customfabric.html (accessed on 10 October 2020).

7. Cornell University-Fabrics of Our Livelihoods. Available online http://smallfarms.cornell.edu/ 2011/07/04/fabrics-of-our-livelihoods/ (accessed on 10 October 2020).

8. Durable water repellent. Available online http://en.wikipedia.org/wiki/Durable_water_repellent (accessed on 10 October 2020).

9. Vatansever, D.; Siores, E.; Hadimani, R.; Shah, T. Smart Woven Fabrics in Renewable Energy Generation. In Advances in Modern Woven Fabrics Technology; Vassiliadis, S., Ed.; InTech: Rijeka, Croatia, 2011; pp. $23-38$.

10. Baurley, S. Interactive and experiential design in smart textile products and applications. Personal and Ubiquitous Computing, 2014, 8, 274-281. 10.1007/s00779-004-0288-5.

11. Eddiai, A.; Meddad, M.; Farhan, R.; Mazroui, M.; Rguiti, M.; Guyomar, D. Corrigendum to “Using PVDF piezoelectric polymers to maximize power harvested by mechanical structure" [Superlattice Microstruct. 127 (2019) 20-26], Superlattices and Microstructures, 2019 https://doi.org/10.1016/j.spmi.2019.106189.

12. Chakhchaoui, N., Farhan, R., Boutaldat, M., Rouway, M., Eddiai, A., Meddad, M., Hajjaji, A.; Cherkaoui, O.; Boughaleb, Y.; Van Langenhove, L. Piezoelectric $\beta$-polymorph formation of new textiles by surface modification with coating process based on interfacial interaction on the conformational variation of poly (vinylidene fluoride)(PVDF) chains. EPJ Appl. Phys. 2020, 91, 31301. https://doi.org/10.1051/epjap/2020200158.

13. Oumghar, K.; Chakhchaoui, N.; Farhane, R.; Eddiai, A.; Meddad, M.; Cherkaoui, O.; Van Langenhove, L. Enhanced piezoelectric properties of PVdF-HFP/PZT nanocomposite for energy harvesting application, IOP Conf. Ser.: Mater. Sci. Eng. 2020, 827, 012034. https://doi.org/10.1088/1757-899X/827/1/012034

14. Chakhchaoui, N.; Farhan, R.; Eddiai, A.; Meddad, M.; Cherkaoui, O.; Mazroui, M.; Boughaleb, Y.; Van Langenhove, L. (2021). Improvement of the electroactive $\beta$-phase nucleation and piezoelectric properties of PVDF-HFP thin films influenced by TiO2 nanoparticles. Materials Today: Proceedings, 2021, 39, 1148-1152. https://doi.org/10.1016/j.matpr.2020.05.407.

15. Chakhchaoui, N.; Jaouani, H.; Farhan, R.; Eddiai, A.; Meddad, M.; Cherkaoui, O.; Van Langenhove, L. An enhanced power harvesting from woven textile using piezoelectric materials. IOP Conf. Ser.: Mater. Sci. Eng. 2020, 827, 012046. https://doi.org/10.1088/1757-899X/827/1/012046.

16. Farhan, R.; Rguiti, M.; Eddiai, A.; Mazroui, M.; Meddad, M.; Courtois, C. Evaluation of performance of polyamide/lead zirconate titanate composite for energy harvesters and actuators, J. Compos. Mater. 2019, 53, 345-352. https://doi.org/10.1177\%2F0021998318783324.

17. Pacelli, M.; Loriga, G.; Taccini, N.; Paradiso, R. Sensing fabrics for monitoring physiological and biomechanical variables: E-textile solutions. 3rd IEEE/EMBS International Summer School on Medical Devices and Biosensors; Cambridge, MA, USA, 2006; pp. 1-4, doi: 10.1109/ISSMDBS.2006.360082.

18. Huang, C. T.; Shen, C. L.; Tang, C. F.; Chang, S. H. A wearable yarn-based piezo-resistive sensor. Sens. Actuators A: Phys. 2008, 141, 396-403. https://doi.org/10.1016/j.sna.2007.10.069.

19. Farhan, R.; Eddiai, A.; Meddad, M.; Mazroui, M.; Guyomar, D. Electromechanical losses evaluation by energyefficient method using the electrostrictive composites: experiments and modeling, Smart Mat. and Struct. 2019, 28, 035024. https://dx.doi.org/10.1088/1361-665X/aafc5c.

20. Meyer, J.; Lukowicz, P.; Troster, G. Textile pressure sensor for muscle activity and motion detection. In 2006 10th IEEE International Symposium on Wearable Computers, Montreux, Switzerland, 11-14 October, 2006; pp. 6972. https://doi.org/10.1109/ISWC.2006.286346.

21. Eleksen Ltd. Available online, http://www.eleksen.com (accessed on 12 November 2020).

22. Logitech Inc. Available online http://www.logitech.com (accessed on 12 November 2020).

23. Ma, R.; Lee, J.; Choi, D.; Moon, H.; Baik, S. Knitted fabrics made from highly conductive stretchable fibers. Nano letters, 2014, 14, 1944-1951. https://doi.org/10.1021/nl404801t.

24. Takamatsu, S.; Kobayashi, T.; Shibayama, N.; Miyake, K.; Itoh, T. Fabric pressure sensor array fabricated with die-coating and weaving techniques, Sens. Actuat. Phys. 2012, 184, 57-63. http://dx.doi.org/10.1016\%2Fj.sna.2012.06.031.

25. Purohit, V.; Choudhary, R. N. P. Structural, dielectric and impedance properties of lead-free $\mathrm{Bi}\left(\mathrm{Sr}_{0} .{ }_{5} \mathrm{Ti}_{0.5}\right) \mathrm{O}_{3}$ ceramic, Materials Science and Engineering: B, 2019, 243, 30-37. https://doi.org/10.1016/j.mseb.2019.03.017.

26. Hajra, S.; Sahu, M.; Purohit, V.; Mohanta, K.; Panigrahi, B. K.; Deepti, P. L. Room temperature electrical properties and excitation performance of $\mathrm{Al}_{2} \mathrm{O}_{3}$ doped fused silica ceramics, Materials Research Express, 2020, 6, 126325. https://doi.org/10.1088/2053-1591/ab648f.

27. Alluri, N. R.; Raj, N. P. M. J.; Khandelwal, G.; Vivekananthan, V.; Kim, S. J. Aloe vera: A tropical desert plant to harness the mechanical energy by triboelectric and piezoelectric approaches, Nano Energy, 2020, 73, 104767. https://doi.org/10.1016/j.nanoen.2020.104767.

28. Hajra, S.; Sahu, M.; Purohit, V.; Choudhary, R. N. P. Dielectric, conductivity and ferroelectric properties of lead-free electronic ceramic: $0.6 \mathrm{Bi} \quad(\mathrm{Fe} 0.98 \quad \mathrm{Ga} 0.02) \quad \mathrm{O}_{3}-0.4 \mathrm{BaTiO}$. Heliyon, 2019, 5, e01654. https://doi.org/10.1016/j.heliyon.2019.e01654. 
29. S. Hajra, V. Purohit, M. Sahu, Electrical characteristics and conduction mechanism of microwave-sintered $\left(\mathrm{Ba}_{0.8 \mathrm{~S}} \mathrm{r}_{0.2}\right) \quad\left(\mathrm{Zr}_{0.1} \mathrm{Ti}_{0.8 \mathrm{C}} \mathrm{Ce} .1\right) \quad \mathrm{O}_{3}$ electronic $\quad$ ceramics. Indian $\quad \mathrm{J} \quad$ Phys. $94(2020) \quad 175-182$. https://doi.org/10.1007/s12648-019-01471-1.

30. Chakhchaoui, N.; Jaouani, H.; Ennamiri, H.; Eddiai, A.; Hajjaji, A.; Meddad, M.; Van Langenhove, L.; Boughaleb, Y. Modeling and analysis of the effect of substrate on the flexible piezoelectric films for kinetic energy harvesting from textiles, J. of Comp. Mat. 2019, 53, 3349-3361. https://doi.org/10.1177\%2F0021998318808869.

31. Hummers Jr, W. S.; Offeman, R. E. Preparation of graphitic oxide, J. Am. Chem. Soc., 1958, 80, 1339-1339. https://doi.org/10.1021/ja01539a017.

32. Tung, V. C.; Allen, M. J.; Yang, Y.; Kaner, R. B. High-throughput solution processing of large-scale graphene. Nat. Nanotechnol. 2004, 4, 25-9. https://doi.org/10.1038/nnano.2008.329.

33. Niyogi, S.; Bekyarova, E.; Itkis, M. E.; McWilliams, J. L.; Hamon, M. A.; Haddon, R. C. Solution properties of graphite and graphene. J. Am. Chem. Soc. 2006, 128, 7720-21. https://doi.org/10.1021/ja060680r.

34. Hirata, M.; Gotou, T.; Horiuchi, S.; Fujiwara, M.; Ohba, M. Thin-film particles of graphite oxide 1: High-yield synthesis and flexibility of the particles, Carbon. 2004, 42, 2929-2937. https://doi.org/10.1016/j.carbon.2004.07.003.

35. Nakajima, T.; Mabuchi, A.; Hagiwara, R. A new structure model of graphite oxide. Carbon. 1988, 26, 357-361. https://doi.org/10.1016/0008-6223(88)90227-8.

36. Lerf, A.; He, H.; Forster, M.; Klinowski, J. Structure of Graphite Oxide Revisited. J. Phys. Chem. B. 1988, 102, 4477-4482. https://doi.org/10.1021/jp9731821.

37. He, H.; Klinowski, J.; Forster, M.; Lerf, A. A new structural model for graphite oxide, Chem. Phys. Lett. 1998, 287, 53-56. https://doi.org/10.1016/S0009-2614(98)00144-4.

38. Cote, L. J.; Kim, F.; Huang, J. Langmuir-Blodgett assembly of graphite oxide single layers. J. Am. Chem. Soc. 2009, 131, 1043-1049. https://doi.org/10.1021/ja806262m.

39. Cai, D.; Song, M. Preparation of fully exfoliated graphite oxide nanoplatelets in organic solvents J. Mater. Chem. 2007, 17, 3678-3680. https://doi.org/10.1039/B705906I.

40. Paredes, J. I.; Villar-Rodil, S.; Martínez-Alonso, A.; Tascon, J. M. D. Graphene oxide dispersions in organic solvents. Langmuir, 2008, 24, 10560-10564. https://doi.org/10.1021/la801744a.

41. Schniepp, H. C.; Li, J. L.; McAllister, M. J.; Sai, H.; Herrera-Alonso, M.; Adamson, D. H.; Prud'homme, R. K.; Car, R.; Saville, D. A.; Aksay, I. A. functionalized single graphene sheets derived from splitting graphite oxide J. Phys. Chem. B. 2006, 110, 8535-8539. https://doi.org/10.1021/jp060936f.

42. McAllister, M. J.; Li, J. L.; Adamson, D. H.; Schniepp, H. C.; Abdala, A. A.; Liu, J.; Herrera-Alonso, M.; Milius, D. L.; Car, R.; Prud'homme, R. K.; Aksay, I. A. Single sheet functionalized graphene by oxidation and thermal expansion of graphite Chem. Mater. 2007, 19, 4396-4404. https://doi.org/10.1021/cm0630800.

43. Li, D.; Müller, M. B.; Gilje, S.; Kaner, R. B.; Wallace, G. G. Processable aqueous dispersions of graphene nanosheets Nat. Nanotechnol. 2008, 3, 101-105. https://doi.org/10.1038/nnano.2007.45.

44. Sun, X.; Liu, Z.; Welsher, K.; Robinson, J. T.; Goodwin, A.; Zaric, S.; Dai, H. Nano-graphene oxide for cellular imaging and drug delivery, Nano. Res. 2008, 1, 203-212. https://doi.org/10.1007/s12274-008-8021-8

45. Ju, H. M.; Huh, S. H.; Choi, S. H.; Lee, H. L. Structures of thermally and chemically reduced graphene Materials Letters, 2010, 64, 357-360. https://doi.org/10.1016/j.matlet.2009.11.016.

46. Marcano, D. C.; Kosynkin, D. V.; Berlin, J. M.; Sinitskii, A.; Sun, Z.; Slesarev, A.; Tour, J. M. Improved Synthesis of Graphene Oxide. ASC Nano, 2010, 4, 4806-4814. https://doi.org/10.1021/nn1006368.

47. Zhang, M.; Zhao, Y.; Yan, L.; Peltier, R.; Hui, W.; Yao, X.; Cui, X.; Sun, H.; Wang, Z. Interfacial Engineering of Bimetallic Ag/Pt Nanoparticles on Reduced Graphene Oxide Matrix for Enhanced Antimicrobial Activity. ACS Appl. Mater. Interfaces. 2016, 8, 8834- 8840. https://doi.org/10.1021/acsami.6b01396.

48. Zhu, P.; Shen, M.; Xiao, S.; Zhang, D.Experimental study on the reducibility of graphene oxide by hydrazine hydrate, Physica. B. 2011, 406, 498-502. https://doi.org/10.1016/j.physb.2010.11.022.

49. Shahriary, L.; Athawale, A. A. Graphene Oxide Synthesized by Using Modified Hummers Approach. Int. J. of Rene Energy and Enviro. Eng. 2014, 2, 58-63.

50. Bykkam, S.; Rao, V.K.; Chakra, C. H. S.; Thunugunta, T. Synthesis and Characterization of Graphene Oxide and Its Antimicrobial Activity against Klebseilla and Staphylococcus. Int. Journal of Adva. Biot. and Rese. 2013, 4, 142-146. http://dx.doi.org/10.4236/graphene.2017.61001.

51. Lee, C.; Wei, X.; Kysar, J. W.; Hone, J. Measurement of the Elastic Properties and Intrinsic Strength of Monolayer Graphene. Science. 2008, 321, 385-388. 10.1126/science.1157996. https://doi.org/10.1126/science.1157996.

52. Ouadil. B.; Cherkaoui. O.; Safia. M.; Zahouily. M. Surface modification of knit polyester fabric for mechanical, electrical and UV protection properties by coating with graphene oxide, graphene and graphene/silver nanocomposites. Applied Surface Science. 2017, 414, 292-302. https://doi.org/10.1016/j.apsusc.2017.04.068. 
53. Nguyen-Tri, P.; Altiparmak, F.; Nguyen, N.; Tuduri, L.; Ouellet-Plamondon, C. M.; Prud'homme, R. E. Robust superhydrophobic cotton fibers prepared by simple dip-coating approach using chemical and plasma-etching pretreatments. ACS omega, 2019, 4, 7829-7837. https://doi.org/10.1021/acsomega.9b00688.

54. Karbownik, I.; Bucheńska, J.; Lipp-Symonowicz, B.; Wrzosek, H. Studies on the influence of grafting PET fibres with acrylic acid on molecular orientation. Fibres \& Textiles. In Eastern Europe, 2008, 16, 108-111.

55. Parvinzadeh, M.; Moradian, S.; Rashidi, A.; Yazdanshenas, M. E. Surface characterization of polyethylene terephthalate/silica nanocomposites. Applied Surface Science, 2010, 256, 2792-2802. 10.1016/j.apsusc.2009.11.030.

56. Jouault, N.; Nanocomposites Silice/polymère: structure des charges, renforcement mécanique, conformation des chaînes et évolution sous déformation. (Doctoral dissertation, Université de Bretagne Sud), 2009.

57. Oumghar, K.; Chakhchaoui, N.; Farhan, R.; Eddiai, A.; Meddad, M.; Cherkaoui, O.; Van Langenhove, L. Piezoelectric $\beta$-polymorph enhancement in graphene oxide-PLA nanocomposite films. IOP Conf. Ser.: Mater. Sci. Eng. 2020, 948, 012024. https://doi.org/10.1088/1757-899X/948/1/012024.

58. Tang, Z. G.; Black, R. A.; Curran, J. M.; Hunt, J. A.; Rhodes, N. P.; Williams, D. F. Surface properties and biocompatibility of solvent-cast poly [E-caprolactone] films. Biomaterials, 2004, 25, 4741-4748. https://doi.org/10.1016/j.biomaterials.2003.12.003.

59. Song, Y.; Zhao, Z.; Yu, W.; Li, B.; Chen, X. Morphological structures of poly (vinylidene fluoride)/montmorillonite nanocomposites. Sci. China Ser. B: Chem. 2007, 50, 790-796. https://doi.org/10.1007/s11426-007-0079-8.

60. Liu, Y.; Recent progress in Fourier transform infrared (FTIR) spectroscopy study of compositional, structural and physical attributes of developmental cotton fibers. Materials, 2013, 6, 299-313. https://doi.org/10.3390/ma6010299.

61. Chung, C.; Lee, M.; Choe, E. K. Characterization of cotton fabric scouring by FT-IR ATR spectroscopy Carbohydrate. Polymers, 2004, 58, 417-420. https://doi.org/10.1177\%2F0040517518790976.

62. Simončič, B.; Tomšič, B.; Orel, B.; Jerman, I. Sol-gel Technology for Chemical Modification of Textiles. In D. Jocić (Ed.), Surface modification systems for creating stimuli responsiveness of textiles: workshop proceedings. University of Twente. 2010; p.17-34. 10.1007/ s10971-017-4382-3.

63. Ince-Gundz, S.; Alpern, R. A.; Amarer, D.; Crawford, J.; Dolan, B.; Kobylarz, R.; Raveley, M.; Cebe, P. Impact of nanosilicates on poly (vinylidene fluoride) crystal polymorphism: Part 1. Melt-crystallization at high supercooling. Polymer, 2010, 51, 1485-1493. https://www.cheric.org/research/tech/periodicals/doi.php?art_seq=834765.

64. Martins, P.; Lopes, A.; Lanceros-Mendez, S.; Electroactive phases of poly (vinylidene fluoride): Determination, processing and applications. Prog. Polym. Sci. 2010, 39, 683-706. http://dx.doi.org/10.1016/j.progpolymsci.2013.07.006.

65. Vinogradov, A.; Holloway, F. Electro-Mechanical Properties of the Piezoelectric Polymer PVDF. Ferroelectrics, 1999, 226, 169-181. https://doi.org/10.1080/00150199908230298.

66. Lovinger, A. J. Poly(Vinylidene fluoride). In: D. C. Bassett, Ed., Development in Crystalline Polymers, Applied Science Publisher. London. 1982, p. 195-261.

67. Gomes, J.; Nunes, J. S.; Sencadas, V.; Lanceros-Méndez, S. Influence of the $\beta$-phase contentand degree of crystallinity on the piezo- and ferroelectric properties of poly (vinylidene fluoride). Smart Mater. Struct. 2010, 19, 065010. https://doi.org/10.1088/0964-1726/19/6/065010.

68. Gallantree, H. R. Review of transducer applications of polyvinylidene fluoride. IEE Proceedings I-Solid-State and Electron Devices. 1983, 130, 219-244. https://doi.org/10.1049/ip-i-1.1983.0040.

69. Zhang, M.; Zhang, A. Q.; Zhu, B. K. ; Du, C. H. ; Xu, Y. Y. Polymorphism in porous poly (vinylidene fluoride) membranes formed via immersion precipitation process. J. Membr. Sci. 2008, 319, 169-175. https://doi.org/10.1016/j.memsci.2008.03.029.

70. Soin, N.; Shah, T. H.; Anand, S. C.; Geng, J.; Pornwannachai, W.; Mandal, P.; Siores, E. Novel 3-D spacer all fibre piezoelectric textiles for energy harvesting applications. Energy Environ. Sci. 2014, 7, 1670-1979. https://doi.org/10.1039/C3EE43987A.

71. Sharma, T.; Aroom, K.; Naik, S.; Gill, B.; Zhang, J. X.; Flexible thin-film PVDF-TrFE based pressure sensor for smart catheter applications. Ann. Biomed. Eng. 2013, 41, 744-751. https://doi.org/10.1007/s10439-012-0708-z.

72. Polla, D. L.; Francis, L. F. Processing and characterization of piezoelectric materials and integration into microelectromechanical systems. Annu. Rev. Mater. Sci. 1998, 28, 563-597. https://doi.org/10.1146/annurev.matsci.28.1.563.

73. Ballato, A. Piezoelectricity: History and new thrusts. In 1996 IEEE Ultrasonics Symposium. Proceedings. 1996, 1, 575-583. https://doi.org/10.1109/ULTSYM.1996.584046.

74. Chakhchaoui, N.; Jaouani, H.; Farhan, R.; Eddiai, A.; Meddad, M.; Cherkaoui, O.; Van Langenhove, L. An enhanced power harvesting from woven textile using piezoelectric materials. IOP Conf. Ser.: Mater. Sci. Eng. 2020, 827, 012046. https://doi.org/10.1088/1757-899X/827/1/012046. 
75. Gregorio, $\mathrm{R}$; Determination of the $\alpha, \beta$, and $\gamma$ crystalline phases of poly (vinylidene fluoride) films prepared at different conditions. J. Appl. Polym. Sci. 2006, 100, 3272-3279. https://doi.org/10.1002/app.23137.

76. Kim, D.; Hong, S.; Li, D.; Roh, H. S.; Ahn, G.; Kim, J.; No, K.; spring-type piezoelectric energy harvester. RSC Adv. 2013, 3, 3194-3198. https://doi.org/10.1039/C2RA22554A.

77. Cardoso, V. F.; Minas, G.; Costa, C. M.; Tavares, C. J.; Lanceros-Mendez, S. Micro and nanofilms of poly(vinylidene fluoride) with controlled thickness, morphology and electroactive crystalline phase for sensor and actuator applications. Smart Mater. Struct. 2011, 20, 087002. https://doi.org/10.1088/0964-1726/20/8/087002.

78. Li, X.; Wang, Y.; Lu, X.; Xiao, C. Morphology changes of polyvinylidene fluoride membrane under different phase separation mechanisms. J. Membr. Sci. 2008, 320, 477-482. https://doi.org/10.1016/j.memsci.2008.04.033.

79. Bottino, A.; Camera-Roda, G.; Capannelli, G.; Munari, S. The formation of microporous polyvinylidene difluoride membranes by phase-separation. J.Membr. Sci. 1991, 57, 1-20. https://doi.org/10.1016/S03767388(00)81159-X.

80. Branciforti, M. C.; Sencadas, V.; Lanceros-Mendez, S. Gregorio Jr, R. New technique of processing highly oriented poly (vinylidene fluoride) films exclusively in the $\beta$ phase. J. Poly. Sci., Part B: Poly. Phys. 2007, 45, 2793-2801. https://doi.org/10.1002/polb.21239.

81. Wendorff, J. H. Concentration fluctuations in poly (vinylidene fluoride)-poly (methyl methacrylate) mixtures. J. Poly. Sci., olym. Lett. Ed. 1980, 18, 439-444. https://doi.org/10.1002/pol.1980.130180607.

82. Young, T. H.; Young, L. T. H.; Cheng, L. P.; Lin, D. J.; Fane, L.; Chuang, W. Y.; Mechanisms of PVDF membrane formation by immersion-precipitation in soft (1-octanol) and harsh (water) non-solvents. Polymer. 1999, 24, 5315-5323. https://doi.org/10.1016/S0032-3861(98)00747-2.

83. Gregorio, R. J.; Cestari, M. Effect of Crystallization Temperature on the Crystalline Phase Content and Morphology of Poly (vinylidene Fluoride). Journal of Polymer Science, Part B: Polymer Physics. 1994, 32, 859-870. https://doi.org/10.1002/polb.1994.090320509.

84. Buonomenna, M. G.; Macchi, P.; Davoli, M.; Drioli, E. Poly (vinylidene fluoride) membranes by phase inversion: the role casting and coagulation conditions play in their morphology, crystalline structure and properties. J. Eur. Polym. 2017, 43, 1557-1572. https://doi.org/10.1016/j.eurpolymj.2006.12.033.

85. Jiao, M.; Yao, Y.; Pastel, G.; Li, T.; Liang, Z.; Xie, H.; Hu, L. Fly-through synthesis of nanoparticles on textile and paper substrates. Nanoscale. 2019, 11, 6174-6181. https://doi.org/10.1039/C8NR10137J.

86. Yang, J.; Pan, P.; Hua, L.; Feng, X.; Yue, J.; Ge, Y.; Inoue, Y. Effects of Crystallization Temperature of Poly (vinylidene fluoride) on Crystal Modification and Phase Transition of Poly (butylene adipate) in Their Blends: A Novel Approach for Polymorphic Control. J. Phys. Chem. B. 2016, 116, 1265-1272. https://doi.org/10.1021/jp209626x.

87. Yang, J.; Pan, P.; Hua, L.; Feng, X.; Yue, J.; Ge, Y.; Inoue, Y. Effects of crystallization temperature of poly(vinylidene fluoride) on crystal modification and phase transition of poly(butylene adipate) in their blends: a novel approach for polymorphic control. J Phys Chem B. 2012, 116, 1265-72. https://doi.org/10.1021/jp209626x.

88. Kang, S. J.; Park, Y. J.; Sung, J.; Jo, P. S.; Park, C.; Kim, K. J.; Cho, B. O. Spin cast ferroelectric beta poly (vinylidene fluoride) thin films via rapid thermal annealing. Appl. Phys. Lett. 2008, 92, 012921. https://doi.org/10.1063/1.2830701.

89. Gregorio, R.; Ueno, E.M. Effect of crystalline phase, orientation and temperature on the dielectric properties of poly (vinylidene fluoride) (PVDF). Journal of Materials Science. 1999, 34, 4489-4500. https://doi.org/10.1023/A:1004689205706.

90. Sencadas, V.; Gregorio Filho, R.; Lanceros-Mendez, S. Processing and characterization of a novel nonporous poly (vinylidene fluoride) films in the $\beta$ phase. Journal of Non-Crystalline Solids. 2006, 352, 2226-2229. https://doi.org/10.1016/I.JNONCRYSOL.2006.02.052.

91. Begum, S.; Kausar, A.; Ullah, H.; Siddiq, M. Potential of Polyvinylidene Fluoride/Carbon Nanotube Composite in Energy, Electronics, and Membrane Technology: An Overview. Polymer-Plastics Technology and Engineering. 2016, 55, 1949-1970. https://doi.org/10.1080/03602559.2016.1185630.

92. Ansari, S.; Giannelis E. P. Functionalized graphene sheet - Poly (vinylidene fluoride) conductive nanocomposites. J. Polym. Sci. Part B: Polym. Phys. 2009, 47, 888-897. https://doi.org/10.1002/polb.21695.

93. Layek, R. K.; Samanta, S.; Chatterjee, D. P.; Nandi, A. K. Physical and mechanical properties of poly (methyl methacrylate)-functionalized graphene/poly (vinylidine fluoride) nanocomposites: Piezoelectric $\beta$ polymorph formation. Polymer. 2010, 51, 5846-5856. https://doi.org/10.1016/j.polymer.2010.09.067.

94. Stankovich, S.; Dikin, D. A.; Dommett, G. H. B.; Kohlhaas, K.M.; Zimney, E. J.; Stach, E. A.; Piner, R. D.; Nguyen, S. T.; Ruoff, R. S. Graphene-Based Composite Materials. Nature. 2006, 442, $282-286$. https://doi.org/10.1038/nature04969. 
95. Lund, A. Hagström, B. Melt spinning of poly (vinylidene fluoride) fibers and the influence of spinning parameters on $\beta$-phase crystallinity. Journal of applied polymer science. 2010, 116, 2685-2693. https://doi.org/10.1002/app.31789.

96. El Achaby, M.; Arrakhiz, F. Z.; Vaudreuil, S.; Essassi, E. M.; Qaiss, A. Piezoelectric $\beta$-polymorph formation and properties enhancement in graphene oxide-PVDF nanocomposite films. Applied Surface Science. 2012, 258, 7668-7677. http://dx.doi.org/10.1016\%2Fj.apsusc.2012.04.118.

97. Kholkin, A. L.; Taylor D. V.; Setter N. Poling effect on the piezoelectric properties of lead zirconate titanate thin films. In ISAF 1998. Proceedings of the Eleventh IEEE International Symposium on Applications of Ferroelectrics IEEE ( Cat. No. 98CH36245). 24-27 August 1988; pp. 69-72. https://doi.org/10.1109/ISAF.1998.786638.

98. Jahan, N.; Mighri, F.; Rodrigue D.; Ajji, A.; Synergistic improvement of piezoelectric properties of

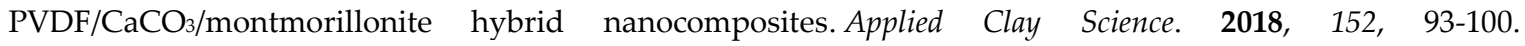
https://doi.org/10.1016/J.CLAY.2017.10.036. 